\title{
Global Robust Exponential Dissipativity for Interval Recurrent Neural Networks with Infinity Distributed Delays
}

\author{
Xiaohong Wang and Huan Qi \\ Department of Control Science and Engineering, Huazhong University of Science and Technology, Wuhan 430074, China \\ Correspondence should be addressed to Xiaohong Wang; wxhong2006@163.com
}

Received 29 January 2013; Accepted 19 May 2013

Academic Editor: Chengming Huang

Copyright ( 2013 X. Wang and H. Qi. This is an open access article distributed under the Creative Commons Attribution License, which permits unrestricted use, distribution, and reproduction in any medium, provided the original work is properly cited.

\begin{abstract}
This paper is concerned with the robust dissipativity problem for interval recurrent neural networks (IRNNs) with general activation functions, and continuous time-varying delay, and infinity distributed time delay. By employing a new differential inequality, constructing two different kinds of Lyapunov functions, and abandoning the limitation on activation functions being bounded, monotonous and differentiable, several sufficient conditions are established to guarantee the global robust exponential dissipativity for the addressed IRNNs in terms of linear matrix inequalities (LMIs) which can be easily checked by LMI Control Toolbox in MATLAB. Furthermore, the specific estimation of positive invariant and global exponential attractive sets of the addressed system is also derived. Compared with the previous literatures, the results obtained in this paper are shown to improve and extend the earlier global dissipativity conclusions. Finally, two numerical examples are provided to demonstrate the potential effectiveness of the proposed results.
\end{abstract}

\section{Introduction}

Neural networks have been a subject of intense research activities over the past few decades due to their wide applications in many areas such as signal processing, pattern recognition, associative memories, parallel computation, and optimization solution. Therefore, increasing attention has been paid to the problem of stability analysis of neural networks with timevarying delays, and recently a lot of research works have been reported for delayed neural networks and system (see [1-17] and references therein).

It is well known that the stability problem is central to analysis of a dynamical system on an equilibrium point. However, from a practical point of view, it is not always the case that the neural network trajectories will approach a single equilibrium point that is the equilibrium point will be unstable. It is also possible that there is no equilibrium point in some situations, especially for interval recurrent neural networks with infinity distributed delays. But what we can know is that the orbits of the neural networks will always enter into a bounded region and stay there from then on. Therefore, the concept of dissipativity (or called Lagrange stability) has been introduced in [18]. Actually, the concept of dissipativity in dynamical systems is a generalization of the Lyapunov stability. The global Lyapunov stability especially can be viewed as a special case of global dissipativity by regarding an equilibrium point as an attractive set [19-21]. Generally speaking, the goal of study on globally dissipative for neural networks is to determine globally attractive sets. Therefore, many initial findings on the global dissipativity $[18,22-30]$ or Lagrange stability [31-36] analysis of neural networks have been reported. At present, global dissipativity theory has been shown to be an appealing and efficient approach for dealing with the problems such as stability theory, chaos and synchronization theory, system norm estimation, and robust control of neural networks without uncertainties $[18,22,23,29,30]$ or of those with uncertainties [24-28, 37, 38].

As it is well known, the use of constant fixed delays in models of delayed feedback provides a good approximation in simple circuits consisting of a small number of cells. However, neural networks usually have a spatial extent due to the presence of a multitude of parallel pathways with a variety of axon sizes and lengths. Thus there will be a distribution of conduction velocities along these pathways and a distribution of propagation delays. In these circumstances, the signal propagation is not instantaneous and cannot be modeled with 
discrete delays. A more appropriate way is to incorporate continuously distributed delays [11, 16, 29, 34, 35, 37, 39-41]. However, these distributed delays are usually unbounded, and this fact motivates our work.

Recently, much attention has also been paid to the robust questions of interval neural networks [7-12, 39, 40, 42, 43]. In [7], global robust stability for stochastic interval neural networks with continuously distributed delays of neutral type has been considered. Bao et al. have investigated the robust stability problem of interval fuzzy Cohen-Grossberg neural networks with piecewise constant argument of generalized type [9]. The global robust passivity analysis for stochastic fuzzy interval neural networks with time-varying delays has been studied in [42]. Balasubramaniam et al. have studied the robust stability for Markovian jumping interval neural networks with discrete and distributed time-varying delays [11]. Xu et al. have studied the stochastic exponential robust stability problem of interval neural networks with reactiondiffusion terms and mixed delays [12]. And the stationary oscillation problem of interval neural networks with discrete and distributed time-varying delays under impulsive perturbations has been studied by using the LMI approach in [40]. Moreover, there are some works on global dissipativity for interval neural networks with time delays such as timevarying delays [24, 25, 27, 38], mixed time-varying delays $[26,37]$. Despite the existence of many reported results in the literature, there are still needs for more in-depth and comprehensive investigations. For example, in almost all the existing results, the activation functions of the neural networks are limited to be sigmoid functions, piecewise linear monotone nondecreasing functions with bounded ranges. Moreover, in these recent publications, time-varying delays [44] terms are required to be continuously differentiable, and the derivative is bounded and smaller than one.

Although there has been published a rich literature on dissipativity problem for neural networks, to the best of our knowledge, few authors pay attention to the dissipativity problem for interval neural networks with both discrete and infinity distributed delays. The problem for global robust exponential dissipativity of IRNNs with mixed time-varying delays and general activation functions, particularly made on it by means of LMIs [2, 6, 27, 36, 38, 40], especially remains open. Hence, this gives the motivation of our present investigation. It is worth pointing out that the proposed results are nontrivial because of (1) establishing a generalized differential inequality which is aimed dealing with the infinity distributed delay appearing in IRNNs; (2) proposing a new Lyapunov-Krasovskii functional that should be used for the general activation function skillfully; (3) proving a lemma to handle the appropriate matrices deformation so as to make use of linear matrix inequalities.

In this paper, we focus on the problem of global robust exponential dissipativity for a class of interval recurrent neural networks with general activation functions and mixed delays, which consists of time-varying and infinite distributed delay. For the sake of comparison, Lyapunov function and Lyapunov-Krasovskii functional are constructed, respectively, which can be used to handle the interval uncertain terms masterly by the LMI approach. What is more, when the
LMIs-based uncertain parameters are feasible, several sufficient conditions are established to guarantee the global robust exponential dissipativity for the addressed IRNNs. And the specific estimation of positive invariant and global exponential attractive sets is also put forward. The purpose of this paper is trebling. First, we demonstrate two important Lemmas which play a vital role in the later theorems. Second, we tackle the problem of global robust exponential dissipativity for IRNNs with both time-varying and infinite distributed delays based on the general activation functions. Third, the results are performed in LMIs, which will be efficiently solved by the MATLAB LMI Toolbox [45] and compared with those presented in $[26,36,37]$. The rest of this paper is organized as follows. In the next section, some preliminaries, including some definitions, assumptions, and significant lemmas, will be described. Section 3 will state the main results. Section 4 will present two illustrative examples to verify the main results, and finally a summery will be given in Section 5 .

Notations. Throughout this paper, $I$ represents the unit matrix; in $R^{+}=[0, \infty)$, the symbols $R^{n}$ and $R^{n \times m}$ stand, respectively, for the $n$-dimensional Euclidean space and the set of all $n \times m$ real matrices. $A^{T}$ and $A^{-1}$ denote the matrix transpose and matrix inverse. $A>0$ or $A<0$ denotes that the matrix $A$ is a symmetric and positive definite or negative definite matrix. Meanwhile, $A<B$ indicates $A-B<0$ and $\|*\|$ is the Euclidean vector norm. When $x$ is a variable, $\|x\|=$ $\sum_{i=1}^{n}\left|x_{i}\right| .[\bullet]^{\dagger}$ denotes the floor function and $\Lambda=\{1,2, \ldots, n\}$. Moreover, in symmetric block matrices, we use an asterisk "*" to represent a term that is induced by symmetry and $\operatorname{diag}\{\cdots\}$ stands for a block-diagonal matrix.

\section{Preliminaries}

The interval recurrent neural networks with infinity distributed delays are described by the following equation group:

$$
\begin{aligned}
\dot{x}(t)= & -D x(t)+A g(x(t))+B g(x(t-\tau(t))) \\
& +C \int_{-\infty}^{t} h(t-s) g(x(s)) d s+U,
\end{aligned}
$$

where $x(t)=\left(x_{1}(t), \ldots, x_{n}(t)\right)^{T}$ is the neuron state vector of the neural network; $U=\left(U_{1}, \ldots, U_{n}\right)^{T}$ is an external input; $\tau(t)$ is the transmission delay of the neural networks, which is time varying and satisfies $0 \leq \tau(t) \leq \tau$, where $\tau$ is a positive constant; $g(x(\cdot))=\left(g_{1}\left(x_{1}(\cdot)\right), \ldots, g_{n}\left(x_{n}(\cdot)\right)\right)^{T}$ represents the neuron activation function, and $h(\cdot)=\operatorname{diag}\left\{h_{1}(\cdot), \ldots, h_{n}(\cdot)\right\}$ represents the delay kernel function. The matrices $D=$ $\operatorname{diag}\left\{d_{1}, \ldots, d_{n}\right\}, A=\left(a_{i j}\right)_{n \times n}, B=\left(b_{i j}\right)_{n \times n}$, and $C=\left(c_{i j}\right)_{n \times n}$ are some unknown diagonal matrix, connection weight matrix, the delayed weight matrix, and the distributively delayed connection weight matrix, respectively, satisfying

$$
D \in \mathbb{D}, \quad A \in \mathbb{A}, \quad B \in \mathbb{B}, \quad C \in \mathbb{C},
$$

where $\mathbb{D}=[\underline{D}, \bar{D}]:=\left\{\operatorname{diag}\left\{d_{1}, \ldots, d_{n}\right\} \mid 0<\underline{d}_{i} \leq d_{i} \leq \bar{d}_{i}, i \in\right.$ $\Lambda\}, \mathbb{A}=[\underline{A}, \bar{A}]:=\left\{\left(a_{i j}\right)_{n \times n} \mid \underline{a}_{i} \leq a_{i} \leq \bar{a}_{i}, i, j \in \Lambda\right\}$, 
$\mathbb{B}=[\underline{B}, \bar{B}]:=\left\{\left(b_{i j}\right)_{n \times n} \mid \underline{b}_{i} \leq b_{i} \leq \bar{b}_{i}, i, j \in \Lambda\right\}, \mathbb{C}=[\underline{C}, \bar{C}]:=$ $\left\{\left(c_{i j}\right)_{n \times n} \mid \underline{c}_{i} \leq c_{i} \leq \bar{c}_{i}, i, j \in \Lambda\right\}$ with $\underline{D}=\operatorname{diag}\left\{\underline{d}_{1}, \ldots, \underline{d}_{n}\right\}$, $\bar{D}=\operatorname{diag}\left\{\bar{d}_{1}, \ldots, \bar{d}_{n}\right\}, \underline{A}=\left(\underline{a}_{i j}\right)_{n \times n}, \bar{A}=\left(\bar{a}_{i j}\right)_{n \times n}, \underline{B}=\left(\underline{a}_{i j}\right)_{n \times n}$, $\bar{B}=\left(\bar{a}_{i j}\right)_{n \times n}, \underline{C}=\left(\underline{a}_{i j}\right)_{n \times n}, \bar{C}=\left(\bar{a}_{i j}\right)_{n \times n}$.

In addition, let

$$
\begin{aligned}
& \Omega:=\left\{\operatorname{diag}\left(\delta_{11}, \ldots, \delta_{1 n}, \ldots, \delta_{n 1}, \ldots, \delta_{n n}\right)\right. \\
& \left.\in R^{n^{2} \times n^{2}}|| \delta_{i j} \mid \leq 1, i, j \in \Lambda\right\}, \\
& D_{0}=\frac{\bar{D}+\underline{D}}{2}, \quad A_{0}=\frac{\bar{A}+\underline{A}}{2} \text {, } \\
& B_{0}=\frac{\bar{B}+\underline{B}}{2}, \quad C_{0}=\frac{\bar{C}+\underline{C}}{2}, \\
& \left(\alpha_{i j}\right)_{n \times n}=\frac{\bar{D}-\underline{D}}{2}, \quad\left(\beta_{i j}\right)_{n \times n}=\frac{\bar{A}-\underline{A}}{2}, \\
& \left(\gamma_{i j}\right)_{n \times n}=\frac{\bar{B}-\underline{B}}{2}, \quad\left(\vartheta_{i j}\right)_{n \times n}=\frac{\bar{C}-\underline{C}}{2}, \\
& M_{1}=\left[\sqrt{\alpha_{11}} e_{1}, \ldots, \sqrt{\alpha_{1 n}} e_{1}, \ldots, \sqrt{\alpha_{n 1}} e_{n}, \ldots, \sqrt{\alpha_{n n}} e_{n}\right]_{n \times n^{2}}, \\
& M_{2}=\left[\sqrt{\beta_{11}} e_{1}, \ldots, \sqrt{\beta_{1 n}} e_{1}, \ldots, \sqrt{\beta_{n 1}} e_{n}, \ldots, \sqrt{\beta_{n n}} e_{n}\right]_{n \times n^{2}} \text {, } \\
& M_{3}=\left[\sqrt{\gamma_{11}} e_{1}, \ldots, \sqrt{\gamma_{1 n}} e_{1}, \ldots, \sqrt{\gamma_{n 1}} e_{n}, \ldots, \sqrt{\gamma_{n n}} e_{n}\right]_{n \times n^{2}} \text {, } \\
& M_{4}=\left[\sqrt{\vartheta_{11}} e_{1}, \ldots, \sqrt{\vartheta_{1 n}} e_{1}, \ldots, \sqrt{\vartheta_{n 1}} e_{n}, \ldots, \sqrt{\vartheta_{n n}} e_{n}\right]_{n \times n^{2}} \text {, } \\
& J_{1}=\left[\sqrt{\alpha_{11}} e_{1}, \ldots, \sqrt{\alpha_{1 n}} e_{n}, \ldots, \sqrt{\alpha_{n 1}} e_{1}, \ldots, \sqrt{\alpha_{n n}} e_{n}\right]_{n^{2} \times n}, \\
& J_{2}=\left[\sqrt{\beta_{11}} e_{1}, \ldots, \sqrt{\beta_{1 n}} e_{n}, \ldots, \sqrt{\beta_{n 1}} e_{1}, \ldots, \sqrt{\beta_{n n}} e_{n}\right]_{n^{2} \times n}, \\
& J_{3}=\left[\sqrt{\gamma_{11}} e_{1}, \ldots, \sqrt{\gamma_{1 n}} e_{n}, \ldots, \sqrt{\gamma_{n 1}} e_{1}, \ldots, \sqrt{\gamma_{n n}} e_{n}\right]_{n^{2} \times n} \text {, } \\
& J_{4}=\left[\sqrt{\vartheta_{11}} e_{1}, \ldots, \sqrt{\vartheta_{1 n}} e_{n}, \ldots, \sqrt{\vartheta_{n 1}} e_{1}, \ldots, \sqrt{\vartheta_{n n}} e_{n}\right]_{n^{2} \times n},
\end{aligned}
$$

where $e_{i} \in R^{n}$ denotes the column vector with $i$ th element to be 1 and others to be 0 .

By some simple calculations, one can transform system (1) into the following form:

$$
\begin{aligned}
\dot{x}(t)= & -\left[D_{0}+M_{1} \Omega_{1} J_{1}\right] x(t)+\left[A_{0}+M_{2} \Omega_{2} J_{2}\right] g(x(t)) \\
& +\left[B_{0}+M_{3} \Omega_{3} J_{3}\right] g(x(t-\tau(t))) \\
& +\left[C_{0}+M_{4} \Omega_{4} J_{4}\right] \int_{-\infty}^{t} h(t-s) g(x(s)) d s+U,
\end{aligned}
$$

Or, equivalently,

$$
\begin{aligned}
\dot{x}(t)= & -D_{0} x(t)+A_{0} g(x(t))+B_{0} g(x(t-\tau(t))) \\
& +C_{0} \int_{-\infty}^{t} h(t-s) g(x(s)) d s+\mathbb{M} \Psi(t)+U,
\end{aligned}
$$

where

$$
\begin{aligned}
\mathbb{M}=\left[M_{1}, M_{2},\right. & \left.M_{3}, M_{4}\right]_{n \times 4 n^{2}}, \quad \Omega_{i} \in \Omega, i=1,2,3,4, \\
\Psi(t)= & \left(\begin{array}{cccc}
\Omega_{1} J_{1} & 0 & 0 & 0 \\
0 & \Omega_{2} J_{2} & 0 & 0 \\
0 & 0 & \Omega_{3} J_{3} & 0 \\
0 & 0 & 0 & \Omega_{4} J_{4}
\end{array}\right) \\
& \times\left(\begin{array}{c}
x(t) \\
g(x(t)) \\
\left.\int_{-\infty}^{t} h(t-\tau(t))\right) \\
h(x-s)
\end{array}\right) .
\end{aligned}
$$

In this paper, the system (1) is supplemented the initial condition given by $x(s)=\phi(s), s \in(-\infty, 0]$, and $i \in \Lambda$, where $\phi(\cdot) \in \mathscr{C}, \mathscr{C}$ denotes real-valued continuous functions defined on $(-\infty, 0]$. Here, it is assumed that, for any initial condition $\phi(\cdot) \in \mathscr{C}$, there exists at least one solution of model (1). As usual, we will also assume that $g(0)=0$ for all $t \in R^{+}$ in this paper.

For further discussion, the following assumptions and lemmas are needed.

(A1) The activation function $g$ satisfies $g(0)=0$, and

$$
l_{j}^{-} \leq \frac{g_{j}(x)-g_{j}(y)}{x-y} \leq l_{j}^{+},
$$

for all $x \neq y, x, y \in R$, where $l_{j}^{+}$and $l_{j}^{-}, j \in \Lambda$, are some real constants.

(A2) The delay kernels $h_{j}(t), j \in \Lambda$ are some real value nonnegative continuous functions defined in $(-\infty, 0]$ and satisfy $h_{j}(t) \leq \mathbb{b}(t), j \in \Lambda$,

$$
\begin{gathered}
\int_{0}^{\infty} h_{j}(t) d t=1, \quad \int_{0}^{\infty} \mathbb{h}(t) d t=\mathbb{h}, \\
\int_{0}^{\infty} \llbracket(t) e^{\varrho t} d t \doteq \mathbb{h}^{\star}<\infty,
\end{gathered}
$$

in which $\mathbb{b}(t)$ corresponds to some nonnegative function defined in $(-\infty, 0]$; constants $\varrho$, $\mathfrak{h}$, and $\mathbb{h}^{\star}{ }^{\star}$ are some positive numbers.

Next, we first introduce the definitions of global robust exponential dissipativity for interval recurrent neural networks (1) or (5) and then state the notation of the upper right Dini derivative and some preliminary lemmas, which are needed to prove our main results.

Definition 1 (see [19]). If there exists a compact set $\Omega \in \mathscr{R}^{n}$ such that, for all $s \in\left[-\infty, t_{0}\right]$, for all $x(s) \in \mathscr{R}^{n} \backslash \Omega$, $\varlimsup_{t \rightarrow+\infty} \rho(x(t), \Omega)=0$; then $\Omega$ is said to be a globally attractive set of (1) or (5), where $\mathscr{R}^{n} \backslash \Omega$ is the complement set of $\Omega$. A set $\Omega$ is called positive invariant set of (1) or (5), if, for all $s \in\left[-\infty, t_{0}\right]$, for all $x(s) \in \mathscr{R}^{n} \backslash \Omega$ implies $x(t) \in \Omega$ for $t \geq t_{0}$.

Definition 2 (see [19]). The neural network defined by (1) or (5) is called a globally exponentially dissipative system, 
if there exists a radially unbounded and positive definite Lyapunov function $V(x(t))$, which satisfies $V(x(t)) \geq\|x\|^{\alpha}$, where $\alpha>0$ is a constant, and constants $\zeta>0, \beta>0$, such that for $V\left(x\left(t_{0}\right)\right)>\zeta, \bar{V}(x(t))>\zeta, t \geq t_{0}$, the inequality $V(x(t))-\zeta \leq\left(\bar{V}\left(x\left(t_{0}\right)\right)-\zeta\right) \exp \left\{-\beta\left(t-t_{0}\right)\right\}$ always holds. And $\{x \mid V(x(t)) \leq \zeta\}$ is said to be a globally exponentially attractive set of (1) or (5), where $\bar{V}\left(x\left(t_{0}\right)\right) \geq V\left(x\left(t_{0}\right)\right)$ and $V\left(x\left(t_{0}\right)\right)$ is a constant.

Definition 3. The neural network defined by (1) or (5) is a globally robustly exponentially dissipative system if the system is globally exponentially dissipative for all $D \in \mathbb{D}, A \in$ $\mathbb{A}, B \in \mathbb{B}$, and $C \in \mathbb{C}$.

Definition 4. For any function $f(t)$, we define its right-hand derivative as

$$
D^{+} f(t)=\lim _{s \rightarrow 0^{+}} \frac{f(t+s)-f(t)}{s} .
$$

Lemma 5 (see [40]). For any vectors $a, b \in R^{n}$, the inequality

$$
\pm 2 a^{T} b \leq a^{T} X a+b^{T} X^{-1} b
$$

holds, in which $X$ is any $n \times n$ matrix with $X>0$.

Lemma 6 (Schur complement [13]). For a given matrix $S=$ $\left(\begin{array}{ll}S_{11} & S_{12} \\ S_{12}^{T} & S_{22}\end{array}\right)$, with $S_{11}=S_{11}^{T}, S_{22}=S_{22}^{T}$, then the following conditions are equivalent:

(1) $S<0$,

(2) $S_{22}<0, S_{11}-S_{12} S_{22}^{-1} S_{12}^{T}<0$,

(3) $S_{11}<0, S_{22}-S_{12}^{T} S_{11}^{-1} S_{12}<0$.

The following two lemmas will be used for deriving our main results.

Lemma 7. Let $p, q, r$, and $\tau$ denote nonnegative constants, and function $f \in C\left(R, R^{+}\right)$satisfies the scalar differential inequality

$$
\begin{aligned}
D^{+} f(t) \leq & -p f(t)+q \sup _{t-\tau \leq s \leq t} f(s) \\
& +r \int_{0}^{\sigma} k(s) f(t-s) d s, \quad t \geq t_{0},
\end{aligned}
$$

where $0<\sigma \leq+\infty, k(\cdot) \in C\left([0, \sigma], R^{+}\right)$satisfies $\int_{0}^{\sigma} k(s) e^{\eta_{0} s} d s$ $<\infty$ for some positive constant $\eta_{0}>0$ in the case when $\sigma=$ $+\infty$. Moreover, when $\sigma=+\infty$, the interval $[t-\sigma, t]$ is understood to be replaced by $(-\infty, t]$. Assume that

$$
p>q+r \int_{0}^{\sigma} k(s) d s .
$$

Then $f(t) \leq \bar{f}\left(t_{0}\right) \exp \left(-\lambda\left(t-t_{0}\right)\right)$ for all $t \geq t_{0}$, where $\bar{f}\left(t_{0}\right)=$ $\sup _{t_{0}-\max \{\sigma, \tau\} \leq s \leq t_{0}} f(s)$, and $\lambda \in\left(0, \eta_{0}\right)$ satisfies the inequality

$$
\lambda<p-q e^{\lambda \tau}-r \int_{0}^{\sigma} k(s) e^{\lambda s} d s .
$$

Proof. We first note that condition (11) implies that there exists a scalar $\lambda \in\left(0, \eta_{0}\right)$ such that inequality (12) holds.

Consider the following equation:

$$
Q(\gamma)=p-q e^{\gamma \tau}-r \int_{0}^{\sigma} k(s) e^{\gamma s} d s-\gamma
$$

Because $Q(0)=p-q-r \int_{0}^{\sigma} k(s) d s>0$ and $Q^{\prime}(\gamma)=-q \tau e^{\gamma \tau}$ $-r \int_{0}^{\sigma} s k(s) e^{\gamma s} d s-1<0$, we follow that $Q(\gamma)$ is a strictly monotone decreasing function. Meanwhile, we also notice that there always exists a positive constant $\eta_{0}$ such that $Q\left(\eta_{0}\right)=p-q \tau e^{\eta_{0} \tau}-r \int_{0}^{\sigma} k(s) e^{\eta_{0} s} d s-\eta_{0}<0$. Therefore, by view of the mean value theorem, there is a constant $0<\lambda^{*}<\eta_{0}$ such that $Q\left(\lambda^{*}\right)=0$. Correspondingly, there exists a constant $0<\lambda<\lambda^{*}<\eta_{0}$ such that $Q(\lambda)>0$, namely, $\lambda<p-q e^{\lambda \tau}$ $-r \int_{0}^{\sigma} k(s) e^{\lambda s} d s$.

Next, we will show $f(t) \leq \bar{f}\left(t_{0}\right) \exp \left(-\lambda\left(t-t_{0}\right)\right)$ for all $t \geq t_{0}$. In order to do this, let

$$
\Psi(t)= \begin{cases}f(t) e^{\lambda\left(t-t_{0}\right)}, & t \geq t_{0}, \\ f(t), & t_{0}-\max \{\sigma, \tau\} \leq t \leq t_{0} .\end{cases}
$$

Now we only need to show that $\Psi(t) \leq \bar{f}\left(t_{0}\right), t \geq t_{0}$. It is clear that $\Psi(t) \leq \bar{f}\left(t_{0}\right)$ for $t_{0}-\max \{\sigma, \tau\} \leq t \leq t_{0}$ by the definition of $\bar{f}$. Next, we can prove that $\Psi(t) \leq \bar{f}\left(t_{0}\right)$ for $t \geq t_{0}$. Suppose, on the contrary, that there exist some $t \in\left[t_{0},+\infty\right)$ such $\Psi(t)>\bar{f}\left(t_{0}\right)$. Let $t^{*}=\inf \left\{t \in\left[t_{0},+\infty\right), \Psi(t)>\bar{f}\left(t_{0}\right)\right\}$; then

$$
\begin{gathered}
\Psi\left(t^{*}\right)=\bar{f}\left(t_{0}\right), \quad \Psi(t) \leq \bar{f}\left(t_{0}\right), \\
t \in\left[t_{0}-\max \{\sigma, \tau\}, t^{*}\right], \\
D^{+} \Psi\left(t^{*}\right) \geq 0 .
\end{gathered}
$$

Suppose that $f\left(\theta_{t^{*}}\right)=\sup _{t^{*}-\tau \leq s \leq t^{*}} f(s), \theta_{t^{*}} \in\left[t^{*}-\tau, t^{*}\right]$. Calculating the upper right Dini derivative $D^{+} \Psi(t)$ along the solution of (5), by (12) and (13), we get

$$
\begin{aligned}
&\left.D^{+} \Psi(t)\right|_{t=t^{*}} \\
&=D^{+} f\left(t^{*}\right) e^{\lambda\left(t^{*}-t_{0}\right)}+\lambda f\left(t^{*}\right) e^{\lambda\left(t^{*}-t_{0}\right)} \\
& \leq {\left[-p f\left(t^{*}\right)+q f\left(\theta_{t^{*}}\right)+r \int_{0}^{\sigma} k(s) f\left(t^{*}-s\right) d s\right] e^{\lambda\left(t^{*}-t_{0}\right)} } \\
&+\lambda f\left(t^{*}\right) e^{\lambda\left(t^{*}-t_{0}\right)} \\
& \leq {\left[(\lambda-p) f\left(t^{*}\right)+q f\left(\theta_{t^{*}}\right)\right.} \\
&\left.+r \int_{0}^{\sigma} k(s) f\left(t^{*}-s\right) d s\right] e^{\lambda\left(t^{*}-t_{0}\right)} \\
&<-q e^{\lambda \tau} f\left(t^{*}\right) e^{\lambda\left(t^{*}-t_{0}\right)}+q e^{\lambda\left(t^{*}-\theta_{t^{*}}\right)} f\left(\theta_{t^{*}}\right) e^{\lambda\left(\theta_{\left.t^{*}-t_{0}\right)}\right)} \\
&-r f\left(t^{*}\right) e^{\lambda\left(t^{*}-t_{0}\right)} \int_{0}^{\sigma} k(s) e^{\lambda s} d s \\
&+r \int_{0}^{\sigma} k(s) e^{\lambda s} f\left(t^{*}-s\right) e^{\lambda\left(t^{*}-s-t_{0}\right)} d s
\end{aligned}
$$




$$
\begin{aligned}
\leq & -q e^{\lambda \tau} \Psi\left(t^{*}\right)+q e^{\lambda \tau} \Psi\left(\theta_{t^{*}}\right)-r \Psi\left(t^{*}\right) \int_{0}^{\sigma} k(s) e^{\lambda s} d s \\
& +r \int_{0}^{\sigma} k(s) e^{\lambda s} \Psi\left(t^{*}-s\right) d s \\
\leq & -q e^{\lambda \tau} \Psi\left(t^{*}\right)+q e^{\lambda \tau} \bar{f}\left(t_{0}\right)-r \Psi\left(t^{*}\right) \int_{0}^{\sigma} k(s) e^{\lambda s} d s \\
& +r \bar{f}\left(t_{0}\right) \int_{0}^{\sigma} k(s) e^{\lambda s} d s=0,
\end{aligned}
$$

which contradicts (13). So we have proven $\Psi(t) \leq \bar{f}\left(t_{0}\right)$ for all $t \geq t_{0}$, that is; $f(t) \leq \bar{f}\left(t_{0}\right) \exp \left(-\lambda\left(t-t_{0}\right)\right)$ for all $t \geq t_{0}$, where $\lambda$ satisfies (12). This completes the proof.

Remark 8 . It should be noted that it is only require that the function $k(\cdot)$ satisfies the assumption: $k(\cdot) \in C\left([0, \sigma], R^{+}\right)$is integrable if $\sigma<+\infty$.

Lemma 9. Given constant matrices $A_{1}, A_{2}, A_{3}, A_{4}, B_{1}, B_{2}$, $B_{3}$, and $B_{4} \in R^{n \times n}$ and appropriate reversible matrices $X_{1}, X_{2}$, $X_{3}$, and $X_{4}$, let

$$
\begin{aligned}
\Sigma_{1}= & \left(\begin{array}{l}
A_{1} \\
B_{1}
\end{array}\right) X_{1}^{-1}\left(\begin{array}{l}
A_{1} \\
B_{1}
\end{array}\right)^{T}+\left(\begin{array}{l}
A_{2} \\
B_{2}
\end{array}\right) X_{2}^{-1}\left(\begin{array}{l}
A_{2} \\
B_{2}
\end{array}\right)^{T} \\
& +\left(\begin{array}{l}
A_{3} \\
B_{3}
\end{array}\right) X_{3}^{-1}\left(\begin{array}{l}
A_{3} \\
B_{3}
\end{array}\right)^{T}+\left(\begin{array}{l}
A_{4} \\
B_{4}
\end{array}\right) X_{4}^{-1}\left(\begin{array}{l}
A_{4} \\
B_{4}
\end{array}\right)^{T}, \\
\Sigma_{2}= & \left(\begin{array}{lllll}
A_{1} & A_{2} & A_{3} & A_{4} \\
B_{1} & B_{2} & B_{3} & B_{4}
\end{array}\right)\left(\begin{array}{cccc}
X_{1}^{-1} & 0 & 0 & 0 \\
0 & X_{2}^{-1} & 0 & 0 \\
0 & 0 & X_{3}^{-1} & 0 \\
0 & 0 & 0 & X_{4}^{-1}
\end{array}\right) \\
& \times\left(\begin{array}{llll}
A_{1} & A_{2} & A_{3} & A_{4} \\
B_{1} & B_{2} & B_{3} & B_{4}
\end{array}\right)^{T} .
\end{aligned}
$$

Then $\Sigma_{1}=\Sigma_{2}$.

Proof. Firstly, we discuss $\Sigma_{1}$, and

$$
\begin{aligned}
\Sigma_{1}= & \left(\begin{array}{cc}
A_{1} X_{1}^{-1} A_{1}^{T} & A_{1} X_{1}^{-1} B_{1}^{T} \\
B_{1} X_{1}^{-1} A_{1}^{T} & B_{1} X_{1}^{-1} B_{1}^{T}
\end{array}\right) \\
& +\left(\begin{array}{cc}
A_{2} X_{2}^{-1} A_{2}^{T} & A_{2} X_{2}^{-1} B_{2}^{T} \\
B_{2} X_{2}^{-1} A_{2}^{T} & B_{2} X_{2}^{-1} B_{2}^{T}
\end{array}\right) \\
& +\left(\begin{array}{cc}
A_{3} X_{3}^{-1} A_{3}^{T} & A_{3} X_{3}^{-1} B_{3}^{T} \\
B_{3} X_{3}^{-1} A_{3}^{T} & B_{3} X_{3}^{-1} B_{3}^{T}
\end{array}\right) \\
& +\left(\begin{array}{cc}
A_{4} X_{4}^{-1} A_{4}^{T} & A_{4} X_{4}^{-1} B_{4}^{T} \\
B_{4} X_{4}^{-1} A_{4}^{T} & B_{4} X_{4}^{-1} B_{4}^{T}
\end{array}\right) \\
= & \left(\begin{array}{cc}
\Delta_{11} & \Delta_{12} \\
* & \Delta_{22}
\end{array}\right),
\end{aligned}
$$

where $\Delta_{11}=A_{1} X_{1}^{-1} A_{1}^{T}+A_{2} X_{2}^{-1} A_{2}^{T}+A_{3} X_{3}^{-1} A_{3}^{T}+A_{4} X_{4}^{-1} A_{4}^{T}$; $\Delta_{12}=A_{1} X_{1}^{-1} B_{1}^{T}+A_{2} X_{2}^{-1} B_{2}^{T}+A_{3} X_{3}^{-1} B_{3}^{T}+A_{4} X_{4}^{-1} B_{4}^{T} ; \Delta_{22}=$ $B_{1} X_{1}^{-1} B_{1}^{T}+B_{2} X_{2}^{-1} B_{2}^{T}+B_{3} X_{3}^{-1} B_{3}^{T}+B_{4} X_{4}^{-1} B_{4}^{T}$.

Calculating $\Sigma_{2}$, we obtain

$$
\begin{aligned}
& \Sigma_{2}=\left(\begin{array}{cccc}
A_{1} X_{1}^{-1} & A_{2} X_{2}^{-1} & A_{3} X_{3}^{-1} & A_{4} X_{4}^{-1} \\
B_{1} X_{1}^{-1} & B_{2} X_{2}^{-1} & B_{3} X_{3}^{-1} & B_{4} X_{4}^{-1}
\end{array}\right) \\
& \times\left(\begin{array}{cc}
A_{1}^{T} & B_{1}^{T} \\
A_{2}^{T} & B_{2}^{T} \\
A_{3}^{T} & B_{3}^{T} \\
A_{4}^{T} & B_{4}^{T}
\end{array}\right) \\
& =\left(\begin{array}{cc}
\Delta_{11} & \Delta_{12} \\
* & \Delta_{22}
\end{array}\right) .
\end{aligned}
$$

Comparing the above equations, we can know $\Sigma_{1}=\Sigma_{2}$. The proof is finished.

Lemma 10 (see [46]). The following inequality is true:

$$
0 \leq \int_{0}^{x_{i}(t)}\left(g_{i}(s)-l_{i}^{-} s\right) d s \leq\left(g_{i}\left(x_{i}(t)\right)-l_{i}^{-} x_{i}(t)\right) x_{i}(t) .
$$

\section{Main Results}

In this section, we shall derive some sufficient conditions for globally robust exponentially dissipative for the IRNNs (1) or (5) with general activation functions, continuous timevarying delay, and infinity distributed time delay by means of applying the lemmas in Section 2 repeatedly.

3.1. Results Using Lyapunov Functions. In this part, sufficient conditions for global robust exponential dissipativity of (1) or (5) are got by using Lyapunov functions and inequality techniques.

Theorem 11. Assume that Assumptions (A1)-(A2) hold; if there exist three constants $\beta_{i}>0, i=1,2$, and 3 , seven positive diagonal matrices $Q_{1}, Q_{2}$, and $Q_{3} \in R^{n \times n}, Q_{4}, Q_{5}, Q_{6}$, and $Q_{7} \in$ $R^{n^{2} \times n^{2}}$, and two positive definite matrices $P, Q_{8} \in R^{n \times n}$ such that the following inequalities hold:

$$
\begin{gathered}
\left(\begin{array}{cccccc}
\Pi_{1} & P A_{0} & P B_{0} & P C_{0} & P M & P \\
* & Q_{1} & 0 & 0 & 0 & 0 \\
* & * & Q_{2} & 0 & 0 & 0 \\
* & * & * & Q_{3} & 0 & 0 \\
* & * & * & * & \Pi_{2} & 0 \\
* & * & * & * & * & Q_{8}
\end{array}\right) \geq 0, \\
\beta_{2} P \geq W Q_{2} W+W J_{3}^{T} Q_{6} J_{3} W \\
\beta_{3} P \geq W Q_{3} W+W J_{4}^{T} Q_{7} J_{4} W, \\
\beta_{1}>\beta_{2}+\beta_{3} \llbracket,
\end{gathered}
$$


where $\Pi_{1}=P D_{0}+D_{0} P-W Q_{1} W-J_{1}^{T} Q_{4} J_{1}-W J_{2}^{T} Q_{5} J_{2} W-$ $\beta_{1} P, W=\operatorname{diag}\left\{w_{1}, \ldots, w_{n}\right\}, w_{j}=\max \left\{\left|l_{j}^{-}\right|,\left|l_{j}^{+}\right|\right\}$,

$$
\Pi_{2}=\left(\begin{array}{cccc}
Q_{4} & 0 & 0 & 0 \\
0 & Q_{5} & 0 & 0 \\
0 & 0 & Q_{6} & 0 \\
0 & 0 & 0 & Q_{7}
\end{array}\right) .
$$

Then the neural network defined by (1) or (5) is a globally robust exponentially dissipative system, and the set $\Phi=\left\{x \in R^{n} \mid\right.$ $\left.x^{T}(t) P x(t) \leq U^{T} Q_{8} U /\left(\beta_{1}-\beta_{2}-\beta_{3} \mathfrak{h}\right)\right\}$ is a positive invariant and globally exponential attractive set.

Proof. Now, we consider the following Lyapunov function:

$$
V(x(t))=x^{T}(t) P x(t) .
$$

Calculating the derivative of $V(x(t))$ along the trajectories of (5), we can obtain

$$
\begin{aligned}
\left.\frac{d V(x(t))}{d t}\right|_{(2)} \leq 2 x^{T}(t) P[ & -D_{0} x(t)+A_{0} g(x(t)) \\
& +B_{0} g(x(t-\tau(t))) \\
& +C_{0} \int_{-\infty}^{t} h(t-s) g(x(s)) d s \\
& +\mathbb{M} \Psi(t)+U]
\end{aligned}
$$

From Assumption (A1) and Lemma 5, we know that there exist three positive diagonal matrices $Q_{1}, Q_{2}$, and $Q_{3} \in R^{n \times n}$ and a positive definite matrix $Q_{8} \in R^{n \times n}$ such that the following inequalities hold:

$$
\begin{aligned}
& 2 x^{T}(t) P A_{0} g(x(t)) \\
& \leq x^{T}(t) P A_{0} Q_{1}^{-1} A_{0}^{T} P x(t)+g^{T}(x(t)) Q_{1} g(x(t)) \\
& \leq x^{T}(t) P A_{0} Q_{1}^{-1} A_{0}^{T} P x(t)+x^{T}(t) W Q_{1} W x(t), \\
& 2 x^{T}(t) P B_{0} g(x(t-\tau(t))) \\
& \leq x^{T}(t) P B_{0} Q_{2}^{-1} B_{0}^{T} P x(t) \\
& \quad+g^{T}(x(t-\tau(t))) Q_{2} g(x(t-\tau(t))) \\
& \leq x^{T}(t) P B_{0} Q_{2}^{-1} B_{0}^{T} P x(t) \\
& \quad+x^{T}(t-\tau(t)) W Q_{2} W x(t-\tau(t)), \\
& 2 x^{T}(t) P C_{0} \int_{-\infty}^{t} h(t-s) g(x(s)) d s \\
& \leq x^{T}(t) P C_{0} Q_{3}^{-1} C_{0}^{T} P x(t)+\left(\int_{-\infty}^{t} h(t-s) g(x(s)) d s\right)^{T} \\
& \quad \times Q_{3}\left(\int_{-\infty}^{t} h(t-s) g(x(s)) d s\right),
\end{aligned}
$$

and by well-known Cauchy-Schwarz inequality and Assumption (A2), we get

$$
\begin{aligned}
& \left(\int_{-\infty}^{t} h(t-s) g(x(s)) d s\right)^{T} Q_{3}\left(\int_{-\infty}^{t} h(t-s) g(x(s)) d s\right) \\
& \quad=\sum_{j=1}^{n} q_{j}^{(3)}\left(\int_{0}^{+\infty} h_{j}(u) g_{j}\left(x_{j}(t-u)\right) d u\right)^{2} \\
& \leq \sum_{j=1}^{n} q_{j}^{(3)} \int_{0}^{+\infty} h_{j}(u) d u \int_{0}^{+\infty} h_{j}(u) g_{j}^{2}\left(x_{j}(t-u)\right) d u \\
& \leq \sum_{j=1}^{n} q_{j}^{(3)} \int_{0}^{+\infty} \mathbb{h}(u) w_{j}^{2} x_{j}^{2}(t-u) d u \\
& \quad=\int_{0}^{+\infty} \mathbb{h}(u) \sum_{j=1}^{n} q_{j}^{(3)} w_{j}^{2} x_{j}^{2}(t-u) d u \\
& \quad=\int_{0}^{+\infty} \mathbb{h}(s) x^{T}(t-s) W Q_{3} W x(t-s) d s,
\end{aligned}
$$

which implies that

$$
\begin{aligned}
2 x^{T}(t) P C_{0} \int_{-\infty}^{t} h(t-s) g(x(s)) d s & \\
\leq & x^{T}(t) P C_{0} Q_{3}^{-1} C_{0}^{T} P x(t) \\
& \quad+\int_{0}^{+\infty} \llbracket(s) x^{T}(t-s) W Q_{3} W x(t-s) d s, \\
& 2 x^{T}(t) P U \leq x^{T}(t) P Q_{8}^{-1} P x(t)+U^{T} Q_{8} U .
\end{aligned}
$$

In view of the definition of $\Omega$, we have the following inequality:

$$
\begin{aligned}
\Psi^{T}(t) \Psi(t) \leq & x^{T}(t) J_{1}^{T} J_{1} x(t)+g^{T}(x(t)) J_{2}^{T} J_{2} g(x(t)) \\
& +g^{T}(x(t-\tau(t))) J_{3}^{T} J_{3} g(x(t-\tau(t))) \\
& +\left(\int_{-\infty}^{t} h(t-s) g(x(s)) d s\right)^{T} J_{4}^{T} J_{4} \\
& \times\left(\int_{-\infty}^{t} h(t-s) g(x(s)) d s\right) .
\end{aligned}
$$

Considering Lemma 5 and (31), we derive

$$
\begin{aligned}
2 x^{T}(t) & P \mathbb{M} \Psi(t) \\
\leq & x^{T}(t) P M_{2}^{-1} \mathbb{M}^{T} P x(t)+\Psi^{T}(t) \Pi_{2} \Psi(t) \\
\leq & x^{T}(t)\left\{P M_{2}^{-1} \mathbb{M}^{T} P+J_{1}^{T} Q_{4} J_{1}+W J_{2}^{T} Q_{5} J_{2} W\right\} x(t) \\
& +x^{T}(t-\tau(t))\left[W J_{3}^{T} Q_{6} J_{3} W\right] x(t-\tau(t)) \\
& +\int_{0}^{+\infty} \llbracket(s) x^{T}(t-s)\left[W J_{4}^{T} Q_{7} J_{4} W\right] x(t-s) d s .
\end{aligned}
$$


Now, adding the terms on the right of (26)-(30) and (32) to (22), considering conditions (17), and making use of Lemma 6, we can obtain that

$$
\begin{gathered}
\left.\frac{d V(x(t))}{d t}\right|_{(2)} \\
\leq x^{T}(t)\left\{-P D_{0}-D_{0} P+P A_{0} Q_{1}^{-1} A_{0}^{T} P+W Q_{1} W\right. \\
\quad+P B_{0} Q_{2}^{-1} B_{0}^{T} P+P C_{0} Q_{3}^{-1} C_{0}^{T} P+P Q_{8}^{-1} P \\
\left.\quad+P M^{-1} M^{T} P+J_{1}^{T} Q_{4} J_{1}+W J_{2}^{T} Q_{5} J_{2} W\right\} x(t) \\
+x^{T}(t-\tau(t))\left[W Q_{2} W+W J_{3}^{T} Q_{6} J_{3} W\right] x(t-\tau(t)) \\
\quad+\int_{0}^{+\infty} \mathbb{h n}(s) x^{T}(t-s)\left[W Q_{3} W+W J_{4}^{T} Q_{7} J_{4} W\right] \\
x(t-s) d s+U^{T} Q_{8} U \\
\leq-\beta_{1} x^{T}(t) P x(t)+\beta_{2} x^{T}(t-\tau(t)) P x(t-\tau(t)) \\
\quad+\beta_{3} \int_{0}^{+\infty} \mathbb{h}(s) x^{T}(t-s) P x(t-s) d s+U^{T} Q_{8} U \\
\leq-\beta_{1} V(x(t))+\beta_{2} V\left(x^{T}(t-\tau(t))\right) \\
+\beta_{3} \int_{0}^{+\infty} \mathbb{h}(s) V(x(t-s)) d s+U^{T} Q_{8} U .
\end{gathered}
$$

Transforming (33) into the following inequality, we get

$$
\begin{aligned}
& \left.\frac{d(V(x(t))-\eta)}{d t}\right|_{(2)} \\
& \leq-\beta_{1}(V(x(t))-\eta)+\beta_{2}\left(\sup _{t-\tau \leq s \leq t} V(x(s))-\eta\right) \\
& \quad+\beta_{3} \int_{0}^{+\infty} \llbracket(s)(V(x(t-s))-\eta) d s, \quad t \geq t_{0},
\end{aligned}
$$

where $\eta=U^{T} H U /\left(\beta_{1}-\beta_{2}-\beta_{3} \mathfrak{h}\right)$.

From formula (34), we can know that it satisfies the (11) of Lemma 7. Meanwhile, noticing Assumption (A2), it can be deduced that $\beta_{1}>\beta_{2}+\beta_{3} \mathbb{h} \Leftrightarrow \beta_{1}>\beta_{2}+\beta_{3} \int_{0}^{+\infty} \mathbb{h}(s) d s$. So (12) of Lemma 7 is also satisfied. From this, when $V(x(t))>$ $\eta$, $\sup _{t-\tau \leq s \leq t} V(x(s))>\eta$, and $\sup _{-\infty \leq s \leq t} V(x(s))>\eta$, according to Lemma 7 , we are able to derive

$$
V(x(t))-\eta \leq(\bar{V}(x(t))-\eta) \exp \left(-\lambda\left(t-t_{0}\right)\right),
$$

where $\bar{V}(x(t))=\sup _{-\infty \leq s \leq t} V(x(s)), \lambda \in(0, \varrho)$ satisfies

$$
\lambda<\beta_{1}-\beta_{2} e^{\lambda \tau}-\beta_{3} \int_{0}^{+\infty} \ln (s) e^{\lambda s} d s .
$$

Simultaneously, judging by [1], it is easy to prove that there exists a constant $\alpha$ such that $V(x(t)) \geq\|x\|^{\alpha}$. In terms of Definitions 1,2 , and 3, we know that the neural network defined by (1) or (5) is a globally robust exponentially dissipative system, and $\Phi=\left\{x \in R^{n} \mid x^{T}(t) P x(t) \leq U^{T} H U /\left(\beta_{1}-\right.\right.$ $\left.\beta_{2}-\beta_{3}(\mathfrak{l})\right\}$ is a positive invariant and globally exponentially attractive set of system (1) or (5). Hence, the proof of Theorem 11 is completed.

Remark 12. It should be noted that the exponential convergence rate $\lambda$ of IRNNs (1) or (5) is also derived in (35). Moreover, one may find that condition $\beta_{1}-\beta_{2}-\beta_{3}$ th $>0$ implies that there exists constant $\lambda \in(0, \varrho)$ such that (1) or (5) holds for any given $\tau>0$.

When $D, A, B$, and $C$ are some known constant matrices, we have the following simple result.

Corollary 13. Assume that Assumptions (A1)-(A2) hold; then the neural network defined by (1) or (5) is a globally exponential dissipative system if there exist three constants $\beta_{i}>0, i=1,2$, and 3, three positive diagonal matrices $Q_{1}, Q_{2}$, and $Q_{3} \in R^{n \times n}$, and two positive definite matrices $P, H \in R^{n \times n}$ such that the following inequalities hold:

$$
\begin{array}{ccccc}
\left(\begin{array}{ccccc}
\Pi_{1} & P A & P B & P C & P \\
* & Q_{1} & 0 & 0 & 0 \\
* & * & Q_{2} & 0 & 0 \\
* & * & * & Q_{3} & 0 \\
* & * & * & * & H
\end{array}\right) \geq 0 \\
\beta_{2} P \geq W Q_{2} W \\
\beta_{3} P \geq W Q_{3} W \\
\beta_{1}>\beta_{2}-\beta_{3} \llbracket
\end{array}
$$

where $\Pi_{1}=P D+D P-W Q_{1} W-P H^{-1} P-\beta_{1} P, W=$ $\operatorname{diag}\left\{w_{1}, \ldots, w_{n}\right\}, w_{j}=\max \left\{\left|l_{j}^{-}\right|,\left|l_{j}^{+}\right|\right\}$, and the set $\Phi=\{x \in$ $\left.R^{n} \mid x^{T}(t) P x(t) \leq U^{T} H U /\left(\beta_{1}-\beta_{2}-\beta_{3} \mathfrak{h}\right)\right\}$ is a positive invariant and globally exponential attractive set.

In the IRNNs system (1), when getting rid of the term of infinite distributed delay $\int_{-\infty}^{t} h(t-s) g(x(s)) d s$, we get Corollary 14 based on Theorem 11.

Corollary 14. Assume that Assumption (A1) holds, if there exist three constants $\beta_{i}>0, i=1,2$, five positive diagonal matrices $Q_{1}, Q_{2} \in R^{n \times n}, Q_{3}, Q_{4}$, and $Q_{5} \in R^{n^{2} \times n^{2}}$, and two positive definite matrices $P, Q_{6} \in R^{n \times n}$ such that the following inequalities hold:

$$
\begin{gathered}
\left(\begin{array}{ccccc}
\Pi_{1} & P A_{0} & P B_{0} & P \mathbb{M} & P \\
* & Q_{1} & 0 & 0 & 0 \\
* & * & Q_{2} & 0 & 0 \\
* & * & * & \Pi_{3} & 0 \\
* & * & * & * & Q_{6}
\end{array}\right) \geq 0 \\
\beta_{2} P \geq W Q_{2} W+W J_{3}^{T} Q_{5} J_{3} W \\
\beta_{1}>\beta_{2},
\end{gathered}
$$


where $\Pi_{1}=P D_{0}+D_{0} P-W Q_{1} W-J_{1}^{T} Q_{3} J_{1}-W J_{2}^{T} Q_{4} J_{2} W-$ $P Q_{6}^{-1} P-\beta_{1} P, W=\operatorname{diag}\left\{w_{1}, \ldots, w_{n}\right\}, w_{j}=\max \left\{\left|l_{j}^{-}\right|,\left|l_{j}^{+}\right|\right\}$,

$$
\Pi_{3}=\left(\begin{array}{ccc}
Q_{3} & 0 & 0 \\
0 & Q_{4} & 0 \\
0 & 0 & Q_{5}
\end{array}\right) .
$$

Then the neural network defined by (1) or (5) is a globally robust exponentially dissipative system, and the set $\Phi=\left\{x \in R^{n} \mid\right.$ $\left.x^{T}(t) P x(t) \leq U^{T} Q_{8} U /\left(\beta_{1}-\beta_{2}\right)\right\}$ is a positive invariant and globally exponential attractive set.

Proof. In front of the course of proof is almost parallel to that of Theorem 11, except for inequality (27) in Theorem 11, here no longer say. In the end, we can also obtain

$$
\begin{aligned}
\left.\frac{d(V(x(t))-\eta)}{d t}\right|_{(1)} \leq & -\beta_{1}(V(x(t))-\eta) \\
& +\beta_{2}(\bar{V}(x(t))-\eta), \quad t \geq t_{0},
\end{aligned}
$$

where $\eta=U^{T} H U /\left(\beta_{1}-\beta_{2}\right), \bar{V}(x(t))=\sup _{t-\tau \leq s \leq t} V(x(s))$.

It is noticed that $\beta_{1}>\beta_{2}$; hence according to the famous Halanay Inequality [47], when $V(x(t))>\eta$ and $\sup _{t-\tau \leq s \leq t} V(x(s))>\eta$, we are able to derive

$$
V(x(t))-\eta \leq(\bar{V}(x(t))-\eta) \exp \left(-\lambda\left(t-t_{0}\right)\right),
$$

where $\lambda$ is the unique positive root of $\lambda=\beta_{1}-\beta_{2} e^{\lambda \tau}$. Similarly, it is obtained that $\Omega=\left\{x \in R^{n} \mid x^{T}(t) P x(t) \leq U^{T} H U /\left(\beta_{1}-\right.\right.$ $\left.\left.\beta_{2}\right)\right\}$ is a positive invariant and globally exponential attractive set of system (1). Hence, the proof is gained.

3.2. Results Using Lyapunov-Krasovskii Functionals. In this part, sufficient conditions for global robust exponential dissipativity of (1) or (5) are obtained by using LyapunovKrasovskii functional and inequality techniques.

Theorem 15. Assume that Assumptions (A1)-(A2) hold, if there exist three constants $\beta_{i}>0, i=1,2$, and 3 , eight positive diagonal matrices $Q, R_{1}, R_{2}$, and $R_{3} \in R^{n \times n}, R_{4}, R_{5}, R_{6}$, and $R_{7} \in$ $R^{n^{2} \times n^{2}}$, and two positive definite matrices $P, R_{8} \in R^{n \times n}$ such that the following inequalities hold:

$$
\Gamma<0,
$$

$$
\begin{gathered}
W R_{2} W+W J_{3}^{T} R_{6} J_{3} W \leq \beta_{2} P, \\
W R_{3} W+W J_{4}^{T} R_{7} J_{4} W \leq \beta_{3} P, \\
\beta_{2}+\beta_{3} \rrbracket_{0}<1+\beta_{1}, \\
\Gamma=\left(\begin{array}{cccccc}
\Theta_{11} & \Theta_{12} & \Theta_{13} & \Theta_{14} & \Theta_{15} & \Theta_{16} \\
* & \Theta_{22} & Q_{0} & Q C_{0} & Q M & Q \\
* & * & -R_{2} & 0 & 0 & 0 \\
* & * & * & -R_{3} & 0 & 0 \\
* & * & * & * & -\Pi & 0 \\
* & * & * & * & * & -R_{8}
\end{array}\right),
\end{gathered}
$$

where $\Theta_{11}=P+Q(F-L)-P D_{0}-D_{0} P+2 L Q D_{0}+W R_{1} W+$ $J_{1}^{T} R_{4} J_{1}+W J_{2}^{T} R_{5} J_{2} W, \Theta_{12}=P A_{0}-L Q A_{0}-D_{0} Q, \Theta_{13}=P B_{0}-$ $L Q B_{0}, \Theta_{14}=P C_{0}-L Q C_{0}, \Theta_{15}=P \mathbb{M}-L Q M, \Theta_{16}=P-L Q$, $\Theta_{22}=Q A_{0}+A_{0}^{T} Q-R_{1}, W=\operatorname{diag}\left\{w_{1}, w_{2}, \ldots, w_{n}\right\}, w_{j}=$ $\max \left\{\left|l_{j}^{-}\right|,\left|l_{j}^{+}\right|\right\}$, for all $j \in \Lambda, L=\operatorname{diag}\left\{l_{1}^{-}, l_{2}^{-}, \ldots, l_{n}^{-}\right\}, F=$ $\operatorname{diag}\left\{l_{1}^{+}, l_{2}^{+}, \ldots, l_{n}^{+}\right\}$,

$$
\Pi=\left(\begin{array}{cccc}
R_{4} & 0 & 0 & 0 \\
0 & R_{5} & 0 & 0 \\
0 & 0 & R_{6} & 0 \\
0 & 0 & 0 & R_{7}
\end{array}\right) .
$$

Then the neural network defined by (1) or (5) is a globally robust exponentially dissipative system, and the set

$$
\Omega=\left\{x \in R^{n} \mid x^{T}(t) P x(t) \leq \frac{U^{T} R_{8} U}{1+\beta_{1}-\beta_{2}-\beta_{3} \mathbb{h}}\right\}
$$

is a positive invariant and globally exponential attractive set of system (1).

Proof. Now, we consider another Lyapunov functional

$$
V(x(t))=x^{T} P x(t)+2 \sum_{i=1}^{n} q_{i} \int_{0}^{x_{i}(t)}\left(g_{i}(s)-l_{i}^{-} s\right) d s .
$$

Calculating the derivative of $V(x(t))$ along the trajectories of (5) and using Lemma 10, we can obtain

$$
\begin{aligned}
& \left.\frac{d V(x(t))}{d t}\right|_{(2)} \\
& \leq 2 x^{T}(t) P\left[-D_{0} x(t)+A_{0} g(x(t))+\mathbb{M} \Psi(t)\right. \\
& +B_{0} g(x(t-\tau(t))) \\
& \left.+C_{0} \int_{-\infty}^{t} h(t-s) g(x(s)) d s+U\right] \\
& +2(g(x(t))-L x(t))^{T} Q \\
& \times\left[-D_{0} x(t)+A_{0} g(x(t))\right. \\
& +\mathbb{M} \Psi(t)+B_{0} g(x(t-\tau(t))) \\
& \left.+C_{0} \int_{-\infty}^{t} h(t-s) g(x(s)) d s+U\right] \\
& =2\left(x^{T}(t) P+g^{T}(x(t)) Q-x^{T}(t) L Q\right) \\
& \times\left(-D_{0} x(t)+A_{0} g(x(t))\right) \\
& +2\left(x^{T}(t) P+g^{T}(x(t)) Q-x^{T}(t) L Q\right) \\
& \times B_{0} g(x(t-\tau(t))) \\
& +2\left(x^{T}(t) P+g^{T}(x(t)) Q-x^{T}(t) L Q\right) C_{0}
\end{aligned}
$$




$$
\begin{aligned}
& \times \int_{-\infty}^{t} h(t-s) g(x(s)) d s \\
& +2\left(x^{T}(t) P+g^{T}(x(t)) Q-x^{T}(t) L Q\right) \mathbb{M} \Psi(t) \\
& +2\left(x^{T}(t) P+g^{T}(x(t)) Q-x^{T}(t) L Q\right) U
\end{aligned}
$$

From Assumption (A1), for given positive diagonal matrix $R_{1}$ we have

$$
\begin{aligned}
2\left(x^{T}(t) P+g^{T}(x(t)) Q-x^{T}(t) L Q\right) \\
\quad \times\left(-D_{0} x(t)+A_{0} g(x(t))\right) \\
\leq 2 x^{T}(t)\left(-P D_{0}+L Q D_{0}\right) x(t)+2 g^{T}(x(t)) Q A_{0} g(x(t)) \\
\quad+2 x^{T}(t)\left(P A_{0}-L Q A_{0}-Q D_{0}\right) g(x(t)) \\
\quad+x^{T}(t) W R_{1} W x(t)-g^{T}(x(t)) R_{1} g(x(t)) \\
=\left(\begin{array}{c}
x(t) \\
g(x(t))
\end{array}\right)^{T} \Xi_{1}\left(\begin{array}{c}
x(t) \\
g(x(t))
\end{array}\right),
\end{aligned}
$$

where

$$
\Xi_{1}=\left(\begin{array}{cc}
\Xi_{11} & P A_{0}-L Q A_{0}-D_{0} Q \\
* & Q A_{0}+A_{0}^{T} Q-R_{1}
\end{array}\right),
$$

$\Xi_{11}=-P D_{0}-D_{0} P+2 L Q D_{0}+W R_{1} W$.

By using Assumption (A1) and Lemma 5, we know that there exist six positive diagonal matrices $R_{2}, R_{3} \in R^{n \times n}, Q_{4}$, $Q_{5}, Q_{6}$, and $Q_{7} \in R^{n^{2} \times n^{2}}$ and a positive definite matrix $R_{8}$ such that the following inequalities hold:

$$
\begin{aligned}
& 2\left(x^{T}(t) P B_{0}+g^{T}(x(t)) Q B_{0}-x^{T}(t) L Q B_{0}\right) g(x(t-\tau(t))) \\
& \leq\left(x^{T}(t) P B_{0}+g^{T}(x(t)) Q B_{0}-x^{T}(t) L Q B_{0}\right) R_{2}^{-1} \\
& \times\left(x^{T}(t) P B_{0}+g^{T}(x(t)) Q B_{0}-x^{T}(t) L Q B_{0}\right)^{T} \\
& +g^{T}(x(t-\tau(t))) R_{2} g(x(t-\tau(t))) \\
& \leq\left(\begin{array}{c}
x(t) \\
g(x(t))
\end{array}\right)^{T}\left(\begin{array}{c}
(P-L Q) B_{0} \\
Q B_{0}
\end{array}\right) R_{2}^{-1}\left(\begin{array}{c}
(P-L Q) B_{0} \\
Q B_{0}
\end{array}\right)^{T} \\
& \times\left(\begin{array}{c}
x(t) \\
g(x(t))
\end{array}\right)+x^{T}(t-\tau(t)) W R_{2} W x(t-\tau(t)), \\
& 2\left(x^{T}(t) P+g^{T}(x(t)) Q-x^{T}(t) L Q\right) U \\
& \leq U^{T} R_{8} U+\left(x^{T}(t) P+g^{T}(x(t)) Q-x^{T}(t) L Q\right) R_{8}^{-1} \\
& \times\left(x^{T}(t) P+g^{T}(x(t)) Q-x^{T}(t) L Q\right)^{T} \\
& =\left(\begin{array}{c}
x(t) \\
g(x(t))
\end{array}\right)^{T}\left(\begin{array}{c}
P-L Q \\
Q
\end{array}\right) R_{8}^{-1} \\
& \times\left(\begin{array}{c}
P-L Q \\
Q
\end{array}\right)^{T}\left(\begin{array}{c}
x(t) \\
g(x(t))
\end{array}\right)+U^{T} R_{8} U
\end{aligned}
$$

$$
\begin{aligned}
& 2\left(x^{T}(t) P C_{0}+g^{T}(x(t)) Q C_{0}-x^{T}(t) L Q C_{0}\right) \\
& \quad \times \int_{-\infty}^{t} h(t-s) g(x(s)) d s \\
& \leq\left(x^{T}(t) P C_{0}+g^{T}(x(t)) Q C_{0}-x^{T}(t) L Q C_{0}\right) R_{3}^{-1} \\
& \quad \times\left(x^{T}(t) P C_{0}+g^{T}(x(t)) Q C_{0}-x^{T}(t) L Q C_{0}\right)^{T} \\
& \quad+\left(\int_{-\infty}^{t} h(t-s) g(x(s)) d s\right)^{T} R_{3} \\
& \quad \times\left(\int_{-\infty}^{t} h(t-s) g(x(s)) d s\right) .
\end{aligned}
$$

Similarly, by well-known Cauchy-Schwarz inequality and Assumption (A2), we get

$$
\begin{aligned}
& \left(\int_{-\infty}^{t} h(t-s) g(x(s)) d s\right)^{T} R_{3}\left(\int_{-\infty}^{t} h(t-s) g(x(s)) d s\right) \\
& \quad \leq \int_{0}^{+\infty} \mathbb{h}(s) x^{T}(t-s) W R_{3} W x(t-s) d s,
\end{aligned}
$$

which implies that

$$
\begin{aligned}
& 2\left(x^{T}(t) P C_{0}+g^{T}(x(t)) Q C_{0}-x^{T}(t) L Q C_{0}\right) \\
& \times \int_{-\infty}^{t} h(t-s) g(x(s)) d s \\
& \leq\left(\begin{array}{c}
x(t) \\
g(x(t))
\end{array}\right)^{T}\left(\begin{array}{c}
(P-L Q) C_{0} \\
Q C_{0}
\end{array}\right) R_{3}^{-1} \\
& \times\left(\begin{array}{c}
(P-L Q) C_{0} \\
Q C_{0}
\end{array}\right)^{T}\left(\begin{array}{c}
x(t) \\
g(x(t))
\end{array}\right) \\
& +\int_{0}^{+\infty} \llbracket(s) x^{T}(t-s) W R_{3} W x(t-s) d s, \\
& 2\left(x^{T}(t) P+g^{T}(x(t)) Q-x^{T}(t) L Q\right) \mathbb{M} \Psi(t) \\
& \leq\left[\left(x^{T}(t) P+g^{T}(x(t)) Q-x^{T}(t) L Q\right) \mathbb{M}\right]^{T} \Pi^{-1} \\
& \times\left[\left(x^{T}(t) P+g^{T}(x(t)) Q-x^{T}(t) L Q\right) \mathbb{M}\right] \\
& +\Psi(t)^{T} \Pi \Psi(t) \\
& \leq\left(\begin{array}{c}
x(t) \\
g(x(t))
\end{array}\right)^{T}\left(\begin{array}{c}
(P-L Q) \mathbb{M} \\
Q \mathbb{M}
\end{array}\right) \Pi^{-1} \\
& \times\left(\begin{array}{c}
(P-L Q) M \\
Q M
\end{array}\right)^{T}\left(\begin{array}{c}
x(t) \\
g(x(t))
\end{array}\right) \\
& +x^{T}(t) J_{1}^{T} R_{4} J_{1} x(t)+g^{T}(x(t)) J_{2}^{T} R_{5} J_{2} g(x(t)) \\
& +g^{T}(x(t-\tau(t))) J_{3}^{T} R_{6} J_{3} g(x(t-\tau(t)))
\end{aligned}
$$




$$
\begin{aligned}
& +\left(\int_{-\infty}^{t} h(t-s) g(x(s)) d s\right)^{T} J_{4}^{T} Q_{7} J_{4} \\
& \times\left(\int_{-\infty}^{t} h(t-s) g(x(s)) d s\right) \\
& \leq\left(\begin{array}{c}
x(t) \\
g(x(t))
\end{array}\right)^{T}\left(\begin{array}{c}
(P-L Q) \mathbb{M} \\
Q M
\end{array}\right) \Pi^{-1}\left(\begin{array}{c}
(P-L Q) \mathbb{M} \\
Q M
\end{array}\right)^{T} \\
& \times\left(\begin{array}{c}
x(t) \\
g(x(t))
\end{array}\right)+x^{T}(t)\left[J_{1}^{T} R_{4} J_{1}+W J_{2}^{T} R_{5} J_{2} W\right] x(t) \\
& +x^{T}(t-\tau(t))\left[W J_{3}^{T} R_{6} J_{3} W\right] x(t-\tau(t)) \\
& +\int_{0}^{+\infty} \mathbb{h}(s) x^{T}(t-s)\left[W J_{4}^{T} Q_{7} J_{4} W\right] x(t-s) d s .
\end{aligned}
$$

Here, the processing method of the term $\Psi(t)^{T} \Pi \Psi(t)$ is similar to the technique of Theorem 11.

Substituting (47)-(52) in (46) and using Lemma 9, we also have

$$
\begin{aligned}
& \left.\frac{d V(x(t))}{d t}\right|_{(1)} \\
& \leq\left(\begin{array}{c}
x(t) \\
g(x(t))
\end{array}\right)^{T}\left(\widetilde{\Xi}_{1}+\Xi_{2}\right)\left(\begin{array}{c}
x(t) \\
g(x(t))
\end{array}\right) \\
& \quad+x^{T}(t-\tau(t))\left[W R_{2} W+W J_{3}^{T} R_{6} J_{3} W\right] x(t-\tau(t)) \\
& \quad+\int_{0}^{+\infty} \mathbb{h}(s) x^{T}(t-s)\left[W R_{3} W+W J_{4}^{T} R_{7} J_{4} W\right] \\
& \quad \times x(t-s) d s+U^{T} R_{8} U,
\end{aligned}
$$

where

$$
\widetilde{\Xi}_{1}=\left(\begin{array}{cc}
\Xi_{11}+J_{1}^{T} R_{4} J_{1}+W J_{2}^{T} R_{5} J_{2} W & P A_{0}-L Q A_{0}-D_{0} Q \\
* & Q A_{0}+A_{0}^{T} Q-R_{1}
\end{array}\right),
$$

$\Xi_{2}$

$$
\begin{aligned}
& =\left(\begin{array}{cccc}
(P-L Q) B_{0} & (P-L Q) C_{0} & (P-L Q) \mathbb{M} & P-L Q \\
Q B_{0} & Q C_{0} & Q \mathbb{M} & Q
\end{array}\right) \\
& \times\left(\begin{array}{cccc}
R_{2}^{-1} & 0 & 0 & 0 \\
0 & R_{3}^{-1} & 0 & 0 \\
0 & 0 & \Pi^{-1} & 0 \\
0 & 0 & 0 & R_{8}^{-1}
\end{array}\right) \\
& \times\left(\begin{array}{cccc}
(P-L Q) B_{0} & (P-L Q) C_{0} & (P-L Q) \mathbb{M} & P-L Q \\
Q B_{0} & Q C_{0} & Q M & Q
\end{array}\right)^{T} .
\end{aligned}
$$

Following from $\Gamma<0$, there exists $0<\beta_{1} \ll 1$ such that

$$
\left(\begin{array}{cccccc}
\widetilde{\Theta}_{11} & \Theta_{12} & \Theta_{13} & \Theta_{14} & \Theta_{15} & \Theta_{16} \\
* & \Theta_{22} & Q_{0} & Q C_{0} & Q M & Q \\
* & * & -R_{2} & 0 & 0 & 0 \\
* & * & * & -R_{3} & 0 & 0 \\
* & * & * & * & -\Pi & 0 \\
* & * & * & * & * & -R_{8}
\end{array}\right)<0,
$$

where $\widetilde{\Theta}_{11}=\left(1+\beta_{1}\right)(P+Q(F-L))-P D_{0}-D_{0} P+2 L Q D_{0}+$ $W R_{1} W+J_{1}^{T} R_{4} J_{1}$. In the light of Lemma 6 , one gets

$$
\left(\begin{array}{cc}
\widetilde{\Theta}_{11} & \Theta_{12} \\
* & \Theta_{22}
\end{array}\right)+\Xi_{2}<0
$$

Meanwhile, it is noticed that

$$
\left(\begin{array}{cc}
\widetilde{\Theta}_{11} & \Theta_{12} \\
* & \Theta_{22}
\end{array}\right)=\widetilde{\Xi}_{1}+\left(\begin{array}{cc}
\left(1+\beta_{1}\right)(P+Q(F-L)) & 0 \\
0 & 0
\end{array}\right) .
$$

Therefore, it can be deduced that

$$
\widetilde{\Xi}_{1}+\Xi_{2}<\left(\begin{array}{cc}
-\left(1+\beta_{1}\right)(P+Q(F-L)) & 0 \\
0 & 0
\end{array}\right) .
$$

Combining the inequalities $W R_{2} W+W J_{3}^{T} R_{6} J_{3} W \leq \beta_{2} P$, $W R_{3} W+W J_{4}^{T} R_{7} J_{4} W \leq \beta_{3} P$ and formulas (53) and (54), we can derive

$$
\begin{aligned}
\left.\frac{d V(x(t))}{d t}\right|_{(2)} \\
\leq-\left(1+\beta_{1}\right) x^{T}(t)(P+Q(F-L)) x(t) \\
\quad+\beta_{2} x^{T}(t-\tau(t)) P x(t-\tau(t))+U^{T} R_{8} U \\
\quad+\beta_{3} \int_{0}^{+\infty} \mathbb{h}(s) x^{T}(t-s) P x(t-s) d s, \quad t \geq t_{0} .
\end{aligned}
$$

From Assumption (A1) and formula (43), one also gets

$$
x^{T}(t) P x(t) \leq V(x(t)) \leq x^{T}(t)(P+Q(F-L)) x(t) .
$$

Noticing $x^{T}(t-\tau(t)) P x(t-\tau(t)) \leq V(t-\tau(t)) \leq$ $\sup _{t-\tau \leq s \leq t} V(x(s))$ and according to (60) and (61), we obtain

$$
\begin{aligned}
& \left.\frac{d V(x(t))}{d t}\right|_{(2)} \\
& \leq-\left(1+\beta_{1}\right) V(x(t))+\beta_{2} \sup _{t-\tau \leq s \leq t} V(x(s)) \\
& \quad+\beta_{3} \int_{0}^{+\infty} \mathbb{h}(s) V(x(t-s)) d s+U^{T} R_{8} U, \quad t \geq t_{0} .
\end{aligned}
$$


Transforming (62) into the following inequality, we get

$$
\begin{aligned}
& \left.\frac{d(V(x(t))-\eta)}{d t}\right|_{(2)} \\
& \leq-\left(1+\beta_{1}\right)(V(x(t))-\eta) \\
& \quad+\beta_{2}\left(\sup _{t-\tau \leq s \leq t} V(x(s))-\eta\right) \\
& \quad+\beta_{3} \int_{0}^{+\infty} \mathbb{R}(s)(V(x(t-s))-\eta) d s, \quad t \geq t_{0},
\end{aligned}
$$

where $\eta=U^{T} R_{8} U /\left(1+\beta_{1}-\beta_{2}-\beta_{3} \llbracket\right)$.

From formula (63), we can know that it satisfies (11) of Lemma 7. Meanwhile, noticing Assumption (A2), it can be deduced that $1+\beta_{1}>\beta_{2}+\beta_{3} \mathfrak{h} \Leftrightarrow 1+\beta_{1}>\beta_{2}+\beta_{3} \int_{0}^{+\infty} \mathfrak{h}(s) d s$. So (12) of Lemma 7 is also satisfied. From this, according to Lemma 7, when $V(x(t))>\eta$, $\sup _{t-\tau \leq s \leq t} V(x(s))>\eta$, and $\sup _{-\infty \leq s \leq t} V(x(s))>\eta$, we are able to derive

$$
V(x(t))-\eta \leq(\bar{V}(x(t))-\eta) \exp \left(-\lambda_{0}\left(t-t_{0}\right)\right),
$$

where $\bar{V}(x(t))=\sup _{-\infty \leq s \leq t} V(x(s))$, and $\lambda_{0} \in(0, \varrho)$ satisfies

$$
\lambda_{0}<1+\beta_{1}-\beta_{2} e^{\lambda_{0} \tau}-\beta_{3} \int_{0}^{+\infty} \operatorname{Rn}(s) e^{\lambda_{0} s} d s
$$

Simultaneously, judging by [1], it is easy to prove that there exists a constant $\alpha$ such that $V(x(t)) \geq\|x\|^{\alpha}$. In terms of Definitions 1,2 , and 3 , we know that the neural network defined by (1) or (5) is a globally robust exponentially dissipative system. And noticing $V(x(t)) \geq x^{T}(t) P x(t)$, it is said that $\Omega=\{x \in$ $\left.R^{n} \mid x^{T}(t) P x(t) \leq U^{T} R_{8} U /\left(1+\beta_{1}-\beta_{2}-\beta_{3} \mathfrak{h}\right)\right\}$ is a positive invariant and globally exponential attractive set of system (1) or (5). Hence, the proof of Theorem 15 is completed.

Remark 16. For the dissipativity or Lagrange condition given in $[22,25,26,28,31-35,37]$, the time delays are constant delays or time-varying delays that are differentiable such that their derivatives are not greater than one or finite. Note that in this paper we do not impose those restrictions on our timevarying delays, which means that our presented results have wider application range.

In the special condition that $C=0$ and $D, A$, and $B$ are some known constant matrices, the model (1) can be rewritten as follows:

$$
\dot{x}(t)=-D x(t)+A g(x(t))+B g(x(t-\tau(t)))+U .
$$

The other conditions are similar to the model (1). Through Theorem 15, the following corollary can be obtained.

Corollary 17. Assume that Assumptions (A1) holds, if there exist three constants $\beta_{i}>0, i=1,2$, three positive diagonal matrices $Q, R_{1}$, and $R_{2} \in R^{n \times n}$, and two positive definite matrices $P, H \in R^{n \times n}$ such that the following inequalities hold:

$$
\begin{gathered}
\Gamma=\left(\begin{array}{cccc}
\Theta_{11} & \Theta_{12} & \Theta_{13} & \Theta_{14} \\
* & \Theta_{22} & Q B & Q \\
* & * & -R_{2} & 0 \\
* & * & * & -H
\end{array}\right)<0 \\
W R_{2} W \leq \beta_{2} P \\
\beta_{2}<1+\beta_{1}
\end{gathered}
$$

where $\Theta_{11}=P+Q(F-L)-P D-D P+2 L Q D+W R_{1} W, \Theta_{12}=$ $P A-L Q A-D Q, \Theta_{13}=P B-L Q B, \Theta_{14}=P-L Q, \Theta_{22}=Q A+$ $A^{T} Q-R_{1}, W=\operatorname{diag}\left\{w_{1}, \ldots, w_{n}\right\}, w_{j}=\max \left\{\left|l_{1}^{-}\right|,\left|l_{1}^{+}\right|\right\}$, for all $j \in \Lambda, L=\operatorname{diag}\left\{l_{1}^{-}, l_{2}^{-}, \ldots, l_{n}^{-}\right\}, F=\operatorname{diag}\left\{l_{1}^{+}, l_{2}^{+}, \ldots, l_{n}^{+}\right\}$, then the neural network (66) is a globally exponential dissipative system, and the set

$$
\Omega=\left\{x \in R^{n} \mid x^{T}(t) P x(t) \leq \frac{U^{T} H U}{1+\beta_{1}-\beta_{2}}\right\}
$$

is a positive invariant and globally exponentially attractive set of system (66).

Proof. The course of proof is almost parallel to that of Corollary 14 and Theorem 15.

Remark 18. When $\beta_{1}>0, \beta_{2}=1$ in Corollary 17, its result will turn into that of [36] right away.

Remark 19. To the best of our knowledge, few authors have discussed the dissipativity analysis of interval neural networks with general activation functions and infinity distributed delay, and there are few results made on it by LMIs $[2,6,27,36,38,40]$. So, the results of this paper are novel and meaningful. Meanwhile, different from [26, 36, 37], some of the more general results are considered in this paper.

Remark 20. It is not also difficult to find that Lemmas 7 and 9 play a vital roles in the whole paper, especially Lemma 7. The full-text results are obtained based on the case of $\sigma=+\infty$ in Lemma 7. In addition, it is needed to point out that Lemma 7 is also suitable for the RNNs (1) with finite distributed delays $\int_{t-\sigma(t)}^{t} f(x(s)) d s$. Consequently, the results of this text are also suitable for the case of finite distributed delay. So, the conclusions of this paper are more general and valuable than the literature $[26,37]$.

Remark 21. In this paper, the main results are obtained mainly by means of constructing two different kinds of $V(x(t))$, of which the difference is depended on the form of general activation function $g(x(t))$. Generally speaking, Theorem 11 is suitable for the case that $g(x(t))$ is unbounded continuous function and Lurie-type function, and Theorem 15 is adapted that $g(x(t))$ is bounded function and Lipschitz-type function. After a short while, in the part of Example 1, about this will be given elaborate illustration. 


\section{Illustrative Examples}

In this section, two numerical examples are presented to demonstrate the usefulness of the developed methods on the globally robust dissipativity by comparing with the previous results of $[19,26,28]$.

Example 1. Consider the interval neural networks model (1) with the following parameters:

$$
\begin{array}{ll}
\tau(t)=0.3+0.5[\sin (t)]^{\dagger}, \quad U=\left(\begin{array}{ll}
1 & 1
\end{array}\right)^{T}, \\
\bar{D}=\left(\begin{array}{cc}
7.8 & 0 \\
0 & 9.6
\end{array}\right), \quad \underline{D}=\left(\begin{array}{cc}
6.2 & 0 \\
0 & 8.4
\end{array}\right), \\
\bar{A}=\left(\begin{array}{cc}
1.6 & 0.1 \\
0.9 & 1.2
\end{array}\right), \quad \underline{A}=\left(\begin{array}{cc}
0 & -0.5 \\
0.1 & 0
\end{array}\right), \\
\bar{B}=\left(\begin{array}{cc}
2 & 0.4 \\
-0.3 & 1.6
\end{array}\right), \quad \underline{B}=\left(\begin{array}{cc}
0.4 & -0.2 \\
-1.1 & 0.4
\end{array}\right), \\
\bar{C}=\left(\begin{array}{cc}
0.2 & 0.8 \\
0 & 1.2
\end{array}\right), \quad \underline{C}=\left(\begin{array}{cc}
-1.4 & 0.2 \\
-0.8 & 0
\end{array}\right) .
\end{array}
$$

And the delay kernel $h(s)$ is elected as $h_{i}(s)=e^{-s}$ for $s \in$ $[0,+\infty), i=1,2$, and 3 .

In this case, by simple calculation, it can be obtained that

$$
\begin{aligned}
& D_{0}=\frac{\bar{D}+\underline{D}}{2}=\left(\begin{array}{ll}
7 & 0 \\
0 & 9
\end{array}\right), \\
& A_{0}=\frac{\bar{A}+\underline{A}}{2}=\left(\begin{array}{cc}
0.8 & -0.2 \\
0.5 & 0.6
\end{array}\right) \text {, } \\
& B_{0}=\frac{\bar{B}+\underline{B}}{2}=\left(\begin{array}{cc}
1.2 & 0.1 \\
-0.7 & 1
\end{array}\right) \text {, } \\
& C_{0}=\frac{\bar{C}+\underline{C}}{2}=\left(\begin{array}{ll}
-0.6 & 0.5 \\
-0.4 & 0.6
\end{array}\right) \text {, } \\
& \left(\alpha_{i j}\right)_{2 \times 2}=\frac{\bar{D}-\underline{D}}{2}=\left(\begin{array}{cc}
0.8 & 0 \\
0 & 0.6
\end{array}\right) \text {, } \\
& \left(\beta_{i j}\right)_{2 \times 2}=\frac{\bar{A}-\underline{A}}{2}=\left(\begin{array}{ll}
0.8 & 0.3 \\
0.4 & 0.6
\end{array}\right) \text {, } \\
& \left(\gamma_{i j}\right)_{2 \times 2}=\frac{\bar{B}-\underline{B}}{2}=\left(\begin{array}{cc}
0.8 & 0.3 \\
0.4 & 0.6
\end{array}\right), \\
& \left(v_{i j}\right)_{2 \times 2}=\frac{\bar{C}-\underline{C}}{2}=\left(\begin{array}{ll}
0.8 & 0.3 \\
0.4 & 0.6
\end{array}\right) \text {, } \\
& M_{1}=\left[\begin{array}{cccc}
\sqrt{0.8} & 0 & 0 & 0 \\
0 & 0 & 0 & \sqrt{0.6}
\end{array}\right] \\
& M_{2}=M_{3}=M_{4}=\left[\begin{array}{cccc}
\sqrt{0.8} & \sqrt{0.3} & 0 & 0 \\
0 & 0 & \sqrt{0.4} & \sqrt{0.6}
\end{array}\right] \text {, }
\end{aligned}
$$

$$
\begin{gathered}
J_{1}=\left[\begin{array}{cc}
\sqrt{0.8} & 0 \\
0 & 0 \\
0 & 0 \\
0 & \sqrt{0.6}
\end{array}\right], \\
J_{2}=J_{3}=J_{4}=\left[\begin{array}{cc}
\sqrt{0.8} & 0 \\
\sqrt{0.3} & 0 \\
0 & \sqrt{0.4} \\
0 & \sqrt{0.6}
\end{array}\right] .
\end{gathered}
$$

Clearly, th $=1, \tau=0.8$, and we choose $\varrho=0.8<1$. In addition, let $g(x(t))=(1 / 4)(|x+1|-|x-1|)$; the activation function satisfies Assumption (A1) with $L=\operatorname{diag}\{-0.5,-0.5\}$, $F=W=\operatorname{diag}\{0.5,0.5\}$. In this case, we choose $\beta_{1}=1, \beta_{2}=$ 0.5 , and $\beta_{3}=0.4$. Obviously, it satisfies the condition $\beta_{2}+$ $\beta_{3}$ th $<1+\beta_{1}$. Note that $\mathbb{M}=\left[M_{1}, M_{2}, M_{3}, M_{4}\right]$, and solving the LMIs in Theorem 15 using the Matlab LMI Control Toolbox, we obtain the feasible solutions as follows:

$$
\begin{aligned}
& P=\left(\begin{array}{cc}
32.07 & 1.41 \\
1.41 & 51.68
\end{array}\right), \quad Q=\left(\begin{array}{cc}
27.35 & 0 \\
0 & 45.68
\end{array}\right), \\
& R_{1}=\left(\begin{array}{cc}
432.2 & 0 \\
0 & 910.0
\end{array}\right), \quad R_{2}=\left(\begin{array}{cc}
4.878 & 0 \\
0 & 3.413
\end{array}\right), \\
& R_{3}=\left(\begin{array}{ccc}
1.099 & 0 \\
0 & 1.311
\end{array}\right), \\
& R_{5}=\left(\begin{array}{cccc}
55.5 & 0 & 0 & 0 \\
0 & 153.7 & 0 & 0 \\
0 & 0 & 231.6 & 0 \\
0 & 0 & 0 & 155.9
\end{array}\right) \\
& R_{6}=\left(\begin{array}{cccc}
2.73 & 0 & 0 & 0 \\
0 & 18.6 & 0 & 0 \\
0 & 0 & 12.9 & 0 \\
0 & 0 & 0 & 7.13
\end{array}\right) \\
& R_{7}=\left(\begin{array}{cccc}
4.84 & 0 & 0 & 0 \\
0 & 23.31 & 0 & 0 \\
0 & 0 & 14.96 & 0 \\
0 & 0 & 0 & 8.63
\end{array}\right)
\end{aligned}
$$

Hence, the above results show that all the conditions stated in Theorem 15 have been satisfied, and the networks (70) is a globally robust exponentially dissipative system. Moreover, by calculating the eigenvalues of $P$, we gain that the set $\Omega=$ $\left\{x \in R^{n} \mid 31.97 x_{1}^{2}+51.78 x_{2}^{2} \leq U^{T} R_{8} U /\left(1+\beta_{1}-\beta_{2}-\beta_{3} \mathfrak{h}\right)=\right.$ $186.35 / 1.1=169.41\}$ is a positive invariant and globally exponential attractive set of (1).

On the other hand, we can conclude that system (70) is globally robust exponentially dissipative by Theorem 11 .

All other things are being equal; if the activation function $g(x)$ is replaced by $g(x(t))=(1 / 2) \tanh (x(t))$, then it is easy 
to check that the LMIs in Theorem 15 do not have feasible solution in MATLAB. Therefore, Theorem 15 is ineffective in the case. However, it can be deduced that the LMIs in Theorem 11 are feasible, and

$$
\begin{gathered}
P=\left(\begin{array}{cc}
3.907 & -0.044 \\
-0.044 & 5.761
\end{array}\right), \quad Q_{1}=\left(\begin{array}{cc}
24.13 & 0 \\
0 & 44.59
\end{array}\right), \\
Q_{2}=\left(\begin{array}{cc}
1.601 & 0 \\
0 & 1.293
\end{array}\right), \quad Q_{3}=\left(\begin{array}{cc}
0.946 & 0 \\
0 & 1.010
\end{array}\right), \\
Q_{4}=14.672 I_{4 \times 4}, \quad Q_{8}=13.912 I_{2 \times 2}, \\
Q_{5}=\left(\begin{array}{cccc}
29.5 & 0 & 0 & 0 \\
0 & 76.3 & 0 & 0 \\
0 & 0 & 111.2 & 0 \\
0 & 0 & 0 & 74.8
\end{array}\right) \\
Q_{6}=\left(\begin{array}{cccc}
1.137 & 0 & 0 & 0 \\
0 & 3.464 & 0 & 0 \\
0 & 0 & 2.626 & 0 \\
0 & 0 & 0 & 1.923
\end{array}\right) \\
Q_{7}=\left(\begin{array}{cccc}
1.501 & 0 & 0 & 0 \\
0 & 3.464 & 0 & 0 \\
0 & 0 & 2.626 & 0 \\
0 & 0 & 0 & 1.923
\end{array}\right)
\end{gathered}
$$

Thereby, by Theorem 11, we obtain that system (70) with $g(x(t))=(1 / 2) \tanh (x(t))$ is globally robust exponentially dissipative, which implies that Theorem 11 can be applied to the case not covered in Theorem 15.

Remark 22. By virtue of Theorem 3.4 in [28], we have $\bar{A}+$ $\bar{A}^{T}+\left(\left\|B^{*}\right\|_{\infty}+\left\|B^{*}\right\|_{1}+\left\|C^{*}\right\|_{\infty}+\left\|C^{*}\right\|_{1}\right) I=\left(\begin{array}{cc}10.7 & 1 \\ 1 & 9.9\end{array}\right)>0$, which does not satisfy the condition of Theorem 3.4, so the conclusion of [28] is not feeble in this case. It implies that the proposed results in this paper improve and generalize [28].

Remark 23. It should be noted that the exponentially dissipative rate $\lambda$ in Example 1 is also obtained, and it satisfies $\lambda \in(0,0.8)$ and $\lambda<1-0.5 e^{0.8 \lambda}-0.4 \int_{0}^{+\infty} e^{(\lambda-1) s} d s$.

Remark 24. In order to imitate the dynamic behavior of system (1), we choose some parameters in (70) randomly as follows:

$$
\begin{array}{rlr}
D=\left(\begin{array}{cc}
6.4 & 0 \\
0 & 8
\end{array}\right), & A=\left(\begin{array}{cc}
1 & 0.8 \\
0.5 & 1
\end{array}\right), \\
B=\left(\begin{array}{cc}
1.5 & 0.1 \\
-0.8 & 1
\end{array}\right), & C=\left(\begin{array}{cc}
-1 & 0.5 \\
-0.6 & 1
\end{array}\right) .
\end{array}
$$

Figures 1 and 2 depict the state trajectories of system (1) with parameters (70), respectively. Figure 3 depicts the phase plots of system (1) with parameters (70) in the case of $g(x(t))=(1 / 2)(|x+1|-|x-1|)$. These numerical results show that system (1) with parameters (70) is globally robustive exponentially dissipative.

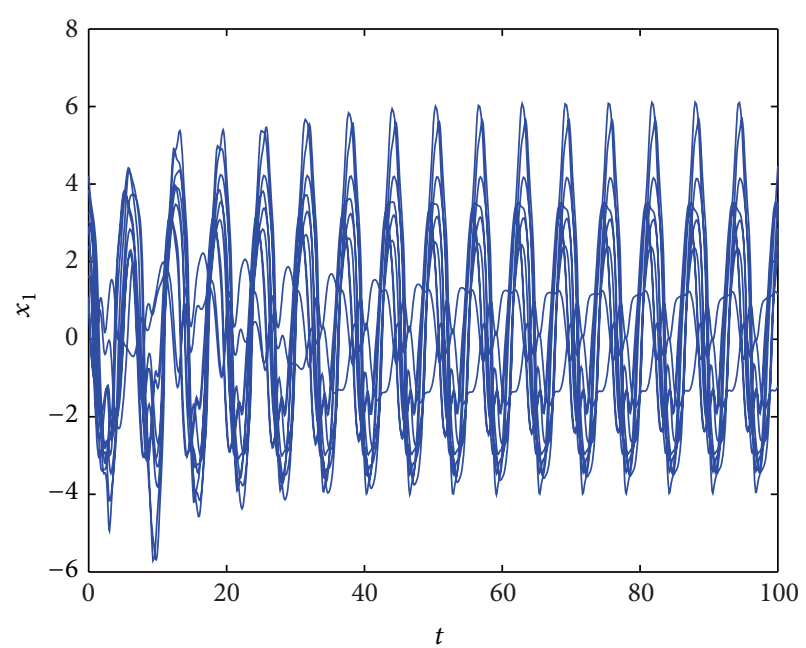

FIGURE 1: The state trajectories of $x_{1}$ in system (70) with arbitrary initial condition.

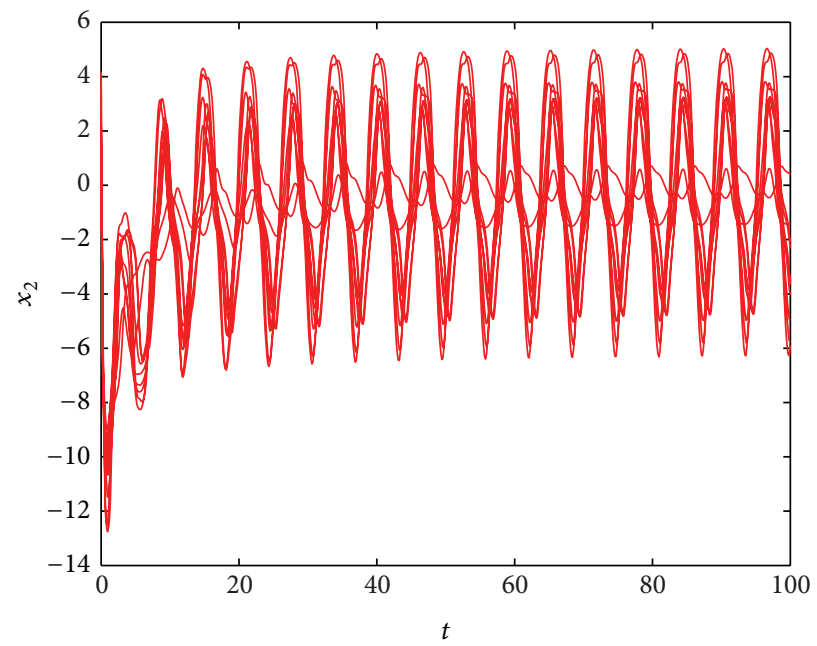

FIGURE 2: The state trajectories of $x_{2}$ in system (70) with arbitrary initial condition.

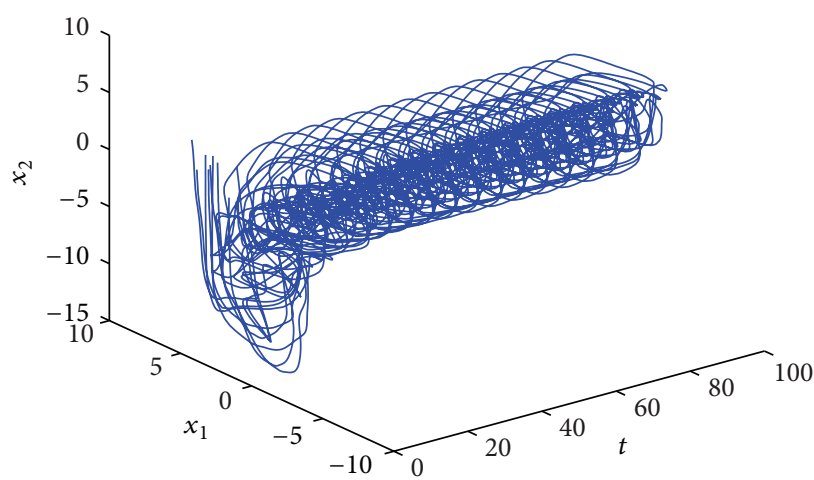

Figure 3: The phase plots of system (70) with random initials. 
Example 2. Considering the following two-neuron RNNs with time-varying delay:

$$
\dot{x}(t)=-D x(t)+A g(x(t))+B g(x(t-\tau(t)))+U,
$$

where $\tau(t)=0.3+0.5[\sin (t)]^{\dagger}, U=(1,1)^{T}$,

$$
\begin{gathered}
D=\left(\begin{array}{cc}
3.5 & 0 \\
0 & 6.2
\end{array}\right), \quad A=\left(\begin{array}{cc}
8 & -2 \\
4 & 2
\end{array}\right), \\
B=\left(\begin{array}{cc}
2 & -5 \\
4 & 8
\end{array}\right) .
\end{gathered}
$$

Clearly, $\tau=0.8$. In addition, If the activation function is chosen as $g(x(t))=(1 / 5)(x(t)+\tanh (x(t)))$, it is obvious that the activation function $g(\cdot)$ satisfies Assumption (A1) with $L=0, F=W=\left(\begin{array}{cc}0.4 & 0 \\ 0 & 0.4\end{array}\right)$.

Under the circumstance, we choose $\beta_{1}=0.5, \beta_{2}=1$. Obviously, it satisfies the condition $\beta_{2}<1+\beta_{1}$. Then by using the MATLAB LMI Control Toolbox, the solutions are derived as follows:

$$
\begin{gathered}
P=\left(\begin{array}{ll}
5.2632 & 0.2579 \\
0.2579 & 9.0456
\end{array}\right), \quad Q=\left(\begin{array}{cc}
1.0160 & 0 \\
0 & 1.7402
\end{array}\right), \\
R_{1}=\left(\begin{array}{cc}
170.0008 & 0 \\
0 & 115.4947
\end{array}\right), \\
R_{2}=\left(\begin{array}{cc}
73.5609 & 0 \\
0 & 143.4947
\end{array}\right), \\
H=\left(\begin{array}{cc}
79.0877 & -0.3404 \\
-0.3404 & 78.1530
\end{array}\right) .
\end{gathered}
$$

Calculating the eigenvalues of $P$, we get the eigenvalues of it are 5.2457 and 9.0631. Therefore, following from Corollary 17, we gain that the set $\Omega=\left\{x \in R^{n} \mid 5.2457 x_{1}^{2}+9.0631 x_{2}^{2} \leq\right.$ $\left.U^{T} H U /\left(1+\beta_{1}-\beta_{2}\right)=156.5599 / 0.5=313.1198\right\}$ is a positive invariant and globally exponential attractive set of system (75). However, according to Theorem 4.2 of [19], we gain

$$
\begin{aligned}
Q^{(1)} & =\left(\begin{array}{ccc}
\frac{A+A^{T}}{2}+I_{2 \times 2}+\operatorname{diag}\left\{-\frac{d_{1}}{w_{1}},-\frac{d_{2}}{w_{2}}\right\} & \frac{B}{2} \\
& \frac{B^{T}}{2} & -I_{2 \times 2}
\end{array}\right) \\
& =\left(\begin{array}{cccc}
0.25 & 1 & 1 & -2.5 \\
1 & -12.5 & 2 & 4 \\
1 & 2 & -1 & 0 \\
-2.5 & 4 & 0 & -1
\end{array}\right) .
\end{aligned}
$$

By calculating we get the four eigenvalues of $Q^{(1)}$ which are $-14.2006,-2.6268,0.0778$, and 2.4997, from which we are able to know that $Q^{(1)}$ is not negative definite. So the conclusion in [19] can not be applied to determine the positive invariant and globally exponential attractive sets of (75) and further ensure the dissipation of system (75).

Meanwhile, noting the conditions of Example 2 also which satisfies Corollary 4 in [26], we verify the effectiveness of its conclusion by means of solving the LMI in equation (34) of [26]. It is easy to check that the linear matrix inequality (34) in [26] has not got feasible solution. Hence, for this example, our results in this paper are less conservative than those in $[19,26]$.

\section{Conclusion}

In this paper, we have studied the globally robust exponentially dissipative for interval neural networks with general activation functions and infinity distributed delays. To the authors best knowledge, few scholars have investigated the globally robust exponentially dissipative for interval neural networks even without infinity distributed delays [26, 37]. By employing the inequality techniques including a novelty delay differential inequality and some LMIs, we have established some sufficient conditions to ensure globally robust exponentially dissipative for interval neural networks with both time-varying delays and infinity distributed delays. In addition, a series of positive invariant and globally exponentially attractive sets of system (1) or (5) are also obtained. Those obtained results improve and complement some recent works (e.g., in $[19,25,26,28,36,37])$. These criteria are stated in LMIs, so that their verification and applications are straightforward and convenient. The results obtained in this paper have also been justified by numerical examples using computer simulations.

For the further work, we intend to generalize the techniques introduced in this paper to the stochastic neural networks based on memristor $[17,48,49]$ or impulsive stochastic reaction-diffusion $[12,14]$ and fuzzy neural networks $[4,15$, $16,50]$.

\section{Acknowledgments}

The authors would like to thank the editor and the anonymous reviewers for their helpful comments and suggestions, which have greatly improved the presentation of the paper.

\section{References}

[1] X. Liao, Y. Fu, and S. Xie, "Globally exponential stability of Hopfied networks," Advances in Systems Science and Applications, vol. 5, pp. 533-545, 2005.

[2] X. Fu and X. Li, "LMI conditions for stability of impulsive stochastic Cohen-Grossberg neural networks with mixed delays," Communications in Nonlinear Science and Numerical Simulation, vol. 16, no. 1, pp. 435-454, 2011.

[3] Q. Zhu, X. Li, and X. Yang, "Exponential stability for stochastic reaction-diffusion BAM neural networks with time-varying and distributed delays," Applied Mathematics and Computation, vol. 217, no. 13, pp. 6078-6091, 2011.

[4] X. Li, R. Rakkiyappan, and P. Balasubramaniam, "Existence and global stability analysis of equilibrium of fuzzy cellular neural networks with time delay in the leakage term under impulsive perturbations," Journal of the Franklin Institute, vol. 348, no. 2, pp. 135-155, 2011. 
[5] D. Li, X. Wang, and D. Xu, "Existence and global p-exponential stability of periodic solution for impulsive stochastic neural networks with delays," Nonlinear Analysis: Hybrid Systems, vol. 6, no. 3, pp. 847-858, 2012.

[6] P. Balasubramaniam and S. Lakshmanan, "LMI conditions for robust stability analysis of stochastic hopfield neural networks with interval time-varying delays and linear fractional uncertainties," Circuits, Systems, and Signal Processing, vol. 30, no. 5, pp. 1011-1028, 2011.

[7] X. Li, "Global robust stability for stochastic interval neural networks with continuously distributed delays of neutral type," Applied Mathematics and Computation, vol. 215, no. 12, pp. 4370-4384, 2010.

[8] W. Han, Y. Kao, and L. Wang, "Global exponential robust stability of static interval neural networks with S-type distributed delays," Journal of the Franklin Institute, vol. 348, no. 8, pp. 2072 2081, 2011.

[9] G. Bao, S. Wen, and Z. Zeng, "Robust stability analysis of interval fuzzy Cohen-Grossberg neural networks with piecewise constant argument of generalized type," Neural Networks, vol. 33, pp. 32-41, 2012.

[10] O. Faydasicok and S. Arik, "Robust stability analysis of a class of neural networks with discrete time delays," Neural Networks, vol. 29-30, pp. 52-59, 2012.

[11] P. Balasubramaniam, S. Lakshmanan, and A. Manivannan, "Robust stability analysis for Markovian jumping interval neural networks with discrete and distributed time-varying delays," Chaos, Solitons and Fractals, vol. 45, no. 4, pp. 483-495, 2012.

[12] X. Xu, J. Zhang, and W. Zhang, "Stochastic exponential robust stability of interval neural networks with reaction-diffusion terms and mixed delays," Communications in Nonlinear Science and Numerical Simulation, vol. 17, no. 12, pp. 4780-4791, 2012.

[13] G. Liu, S. Yang, Y. Chai, W. Feng, and W. Fu, "Robust stability criteria for uncertain stochastic neural networks of neutral-type with interval time-varying delays," Neural Computing and Applications, vol. 22, no. 2, pp. 349-359, 2013.

[14] D. Li, D. He, and D. Xu, "Mean square exponential stability of impulsive stochastic reaction-diffusion Cohen-Grossberg neural networks with delays," Mathematics and Computers in Simulation, vol. 82, no. 8, pp. 1531-1543, 2012.

[15] S. Long, Q. Song, X. Wang, and D. Li, "Stability analysis of fuzzy cellular neural networks with time delay in the leakage term and impulsive perturbations," Journal of the Franklin Institute, vol. 349, no. 7, pp. 2461-2479, 2012.

[16] H. Li, B. Chen, C. Lin, and Q. Zhou, "Mean square exponential stability of stochastic fuzzy Hopfield neural networks with discrete and distributed time-varying delays," Neurocomputing, vol. 72, no. 7-9, pp. 2017-2023, 2009.

[17] S. Wen, Z. Zeng, and T. Huang, "Exponential stability analysis of memristor-based recurrent neural networks with time-varying delays," Neurocompting, vol. 97, pp. 233-240, 2012.

[18] X. Liao and J. Wang, "Global dissipativity of continuous-time recurrent neural networks with time delay," Physical Review E, vol. 68, no. 1, Article ID 016118, 2003.

[19] X. Liao, Q. Luo, and Z. Zeng, "Positive invariant and global exponential attractive sets of neural networks with time-varying delays," Neurocomputing, vol. 71, no. 4-6, pp. 513-518, 2008.

[20] D. He and D. Xu, "Attracting and invariant sets of fuzzy CohenGrossberg neural networks with time-varying delays," Physics Letters A, vol. 372, no. 47, pp. 7057-7062, 2008.
[21] Y. Huang, W. Zhu, and D. Xu, "Invariant and attracting set of fuzzy cellular neural networks with variable delays," Applied Mathematics Letters, vol. 22, no. 4, pp. 478-483, 2009.

[22] Z. G. Yang and Z. C. Yang, "Dissipativity in mean square of nonautonomous impulsive stochastic neural networks with delays," in Proceedings of the 7th International Symposium on Neural Networks (ISNN '10), vol. 6063 of Lecture Notes in Computer Science, pp. 735-744, 2010.

[23] X. Y. Lou and B. T. Cui, "Global robust dissipativity for integrodifferential systems modeling neural networks with delays," Chaos, Solitons and Fractals, vol. 36, no. 2, pp. 469-478, 2008.

[24] M. Luo and S. Zhong, "Global dissipativity of uncertain discrete-time stochastic neural networks with time-varying delays," Neurocomputing, vol. 85, pp. 20-28, 2012.

[25] Q. Song and J. Cao, "Global dissipativity on uncertain discretetime neural networks with time-varying delays," Discrete Dynamics in Nature and Society, vol. 2010, Article ID 810408, 19 pages, 2010.

[26] Q. Song and J. Cao, "Global dissipativity analysis on uncertain neural networks with mixed time-varying delays," Chaos, vol. 18, no. 4, Article ID 043126, 2008.

[27] G. Wang, J. Cao, and L. Wang, "Global dissipativity of stochastic neural networks with time delay," Journal of the Franklin Institute, vol. 346, no. 8, pp. 794-807, 2009.

[28] B. Cui and X. Lou, "Global robust dissipativity of integro-differential systems modelling neural networks with time delay," Electronic Journal of Differential Equations, vol. 2007, no. 89, pp. $1-12,2007$.

[29] Z. Feng and J. Lam, "Stability and dissipativity analysis of distributed delay cellular neural networks," IEEE Transactions on Neural Networks, vol. 22, no. 6, pp. 976-981, 2011.

[30] Z. Wu, J. Lam, H. Su, and J. Chu, "Stability and dissipativity analysis of static neural networks with time delay," IEEE Transactions on Neural Networks and Learning Systems, vol. 23, no. 2, pp. 199-210, 2012.

[31] X. Liao, Q. Luo, Z. Zeng, and Y. Guo, "Global exponential stability in Lagrange sense for recurrent neural networks with time delays," Nonlinear Analysis: Real World Applications, vol. 9, no. 4, pp. 1535-1557, 2008.

[32] Q. Luo, Z. Zeng, and X. Liao, "Global exponential stability in Lagrange sense for neutral type recurrent neural networks," Neurocomputing, vol. 74, no. 4, pp. 638-645, 2011.

[33] A. Wu, Z. Zeng, C. Fu, and W. Shen, "Global exponential stability in Lagrange sense for periodic neural networks with various activation functions," Neurocomputing, vol. 74, no. 5, pp. 831-837, 2011.

[34] X. Wang, M. Jiang, and S. Fang, "Stability analysis in Lagrange sense for a non-autonomous Cohen-Grossberg neural network with mixed delays," Nonlinear Analysis, Theory, Methods and Applications, vol. 70, no. 12, pp. 4294-4306, 2009.

[35] B. Wang, J. Jian, and M. Jiang, "Stability in Lagrange sense for Cohen-Grossberg neural networks with time-varying delays and finite distributed delays," Nonlinear Analysis: Hybrid Systems, vol. 4, no. 1, pp. 65-78, 2010.

[36] Z. Tu, J. Jian, and K. Wang, "Global exponential stability in Lagrange sense for recurrent neural networks with both timevarying delays and general activation functions via LMI approach," Nonlinear Analysis: Real World Applications, vol. 12, no. 4, pp. 2174-2182, 2011.

[37] L. Wang and J. Cao, "Global robust point dissipativity of interval neural networks with mixed time-varying delays," Nonlinear Dynamics, vol. 55, no. 1-2, pp. 169-178, 2009. 
[38] S. Muralisankar, N. Gopalakrishnan, and P. Balasubramaniam, "An LMI approach for global robust dissipativity analysis of T-S fuzzy neural networks with interval time-varying delays," Expert Systems with Applications, vol. 39, no. 3, pp. 3345-3355, 2012.

[39] P. Balasubramaniam, G. Nagamani, and R. Rakkiyappan, "Global passivity analysis of interval neural networks with discrete and distributed delays of neutral type," Neural Processing Letters, vol. 32, no. 2, pp. 109-130, 2010.

[40] X. Li and J. Shen, "LMI approach for stationary oscillation of interval neural networks with discrete and distributed timevarying delays under impulsive perturbations," IEEE Transactions on Neural Networks, vol. 21, no. 10, pp. 1555-1563, 2010.

[41] H. Li, H. Gao, and P. Shi, "New passivity analysis for neural networks with discrete and distributed delays," IEEE Transactions on Neural Networks, vol. 21, no. 11, pp. 1842-1847, 2010.

[42] P. Balasubramaniam and G. Nagamani, "Global robust passivity analysis for stochastic fuzzy interval neural networks with timevarying delays," Expert Systems with Applications, vol. 39, no. 1, pp. 732-742, 2012.

[43] P. Balasubramaniam and G. Nagamani, "A delay decomposition approach to delay-dependent passivity analysis for interval neural networks with time-varying delay," Neurocomputing, vol. 74, no. 10, pp. 1646-1653, 2011.

[44] S. Wen, Z. Zeng, T. Huang, and G. Bao, "Robust passivity and passification for a class of singularly perturbed nonlinear systems with time-varying delays and polytopic uncertainties via neural networks," Circuits, Systems, and Signal Processing, vol. 23, no. 3, pp. 1113-1127, 2013.

[45] P. Gahinet, A. Nemirovski, A. Laub, and M. Chilali, LMI Control Toolbox User's Guide, Mathworks, Natick, Mass, USA, 1995.

[46] D. Zhang and L. Yu, "Exponential state estimation for Markovian jumping neural networks with time-varying discrete and distributed delays," Neural Networks, vol. 35, pp. 103-111, 2012.

[47] A. Halanay, Differential Equations: Stability, Oscillations, Time Lags, Academic Press, New York, NY, USA, 1966.

[48] S. Wen, Z. Zeng, and T. Huang, "Dynamic behaviors of memristor-based delayed recurrent networks," Neural Computing and Applications, 2012.

[49] S. Wen and Z. Zeng, "Dynamics analysis of a class of memristorbased recurrent networks with time-varying delays in the presence of strong external stimuli," Neural Processing Letters, vol. 35, no. 1, pp. 47-59, 2012.

[50] L. Chen, Y. Chai, and R. Wu, "Covergence of stochastic fuzzy Cohen-Grossberg neural networks with reaction-diffusion and distributed delays," Expert Systems with Applications, vol. 39, no. 5, pp. 5767-5773, 2012. 


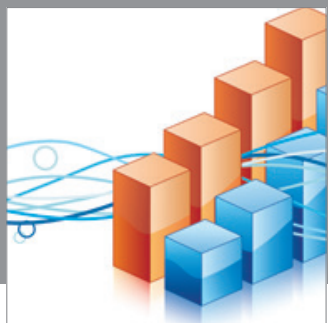

Advances in

Operations Research

mansans

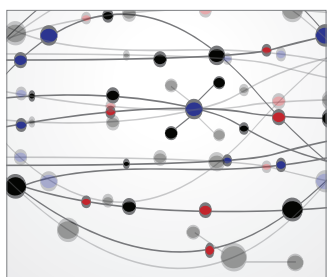

The Scientific World Journal
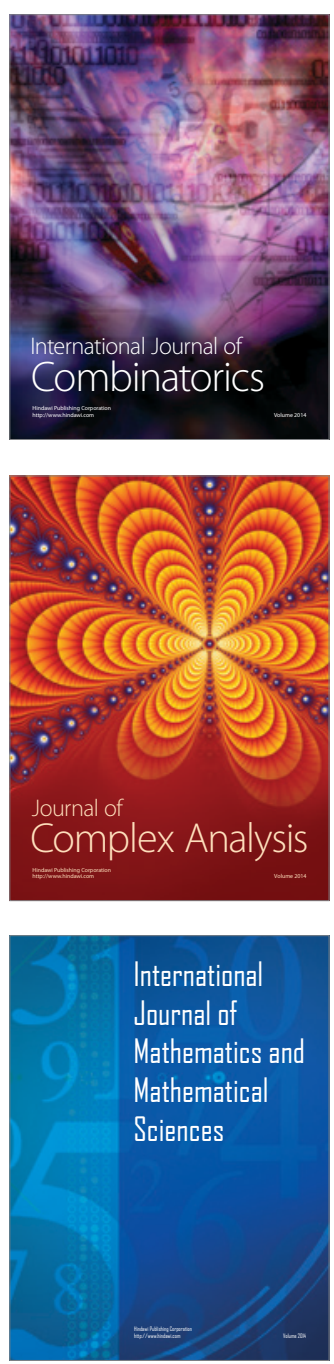
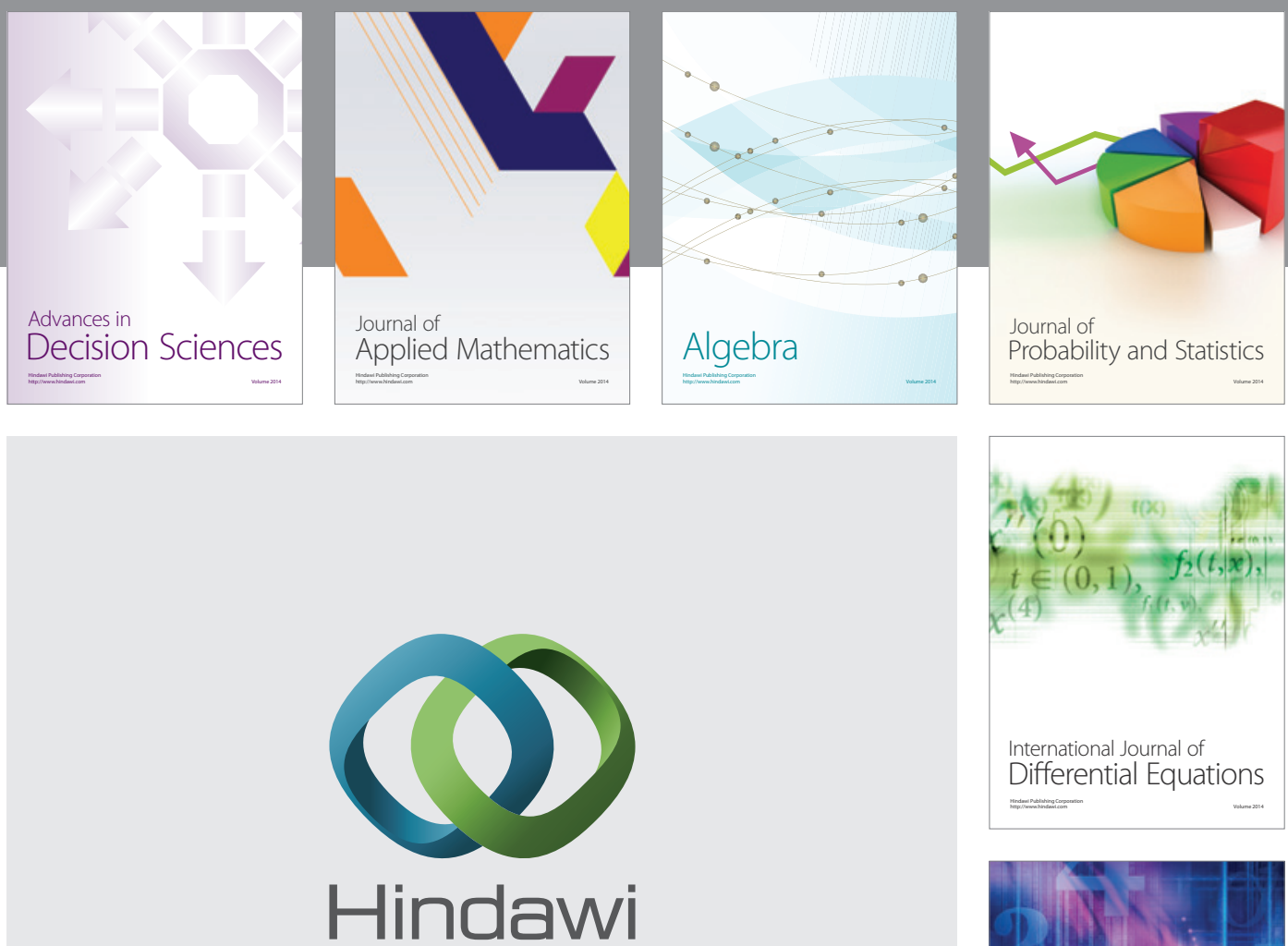

Submit your manuscripts at http://www.hindawi.com
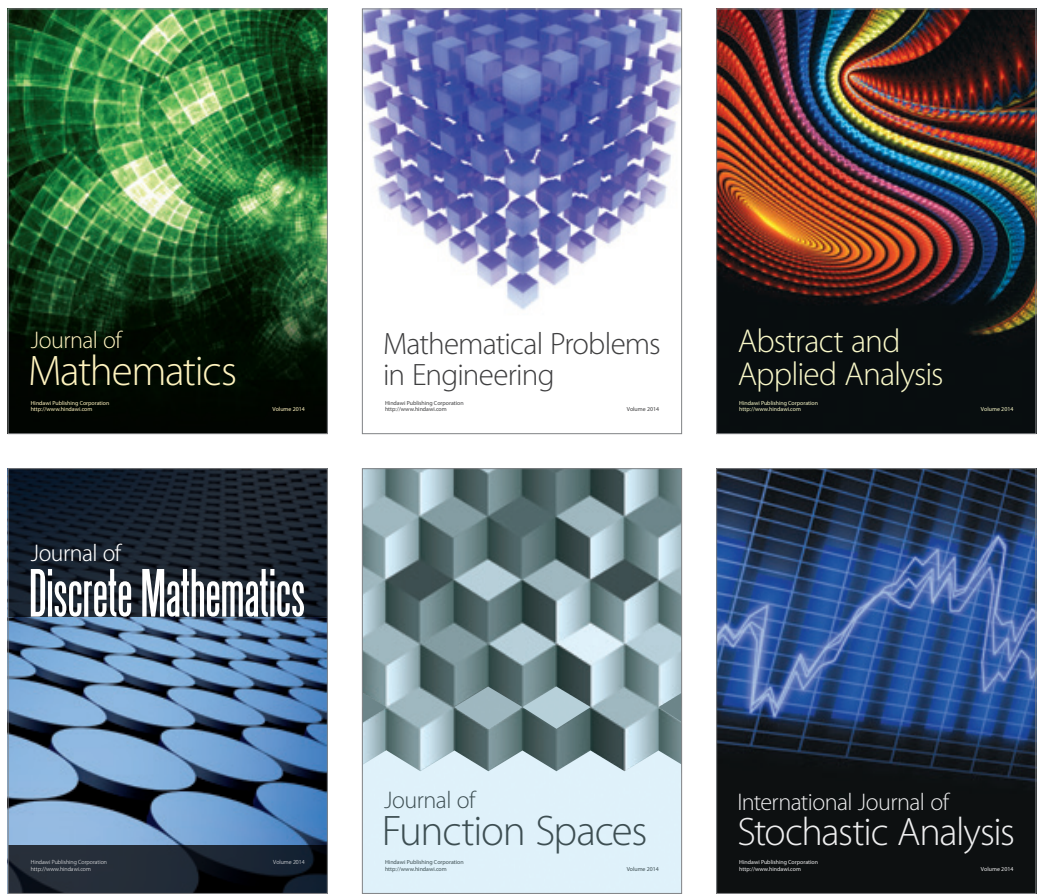

Journal of

Function Spaces

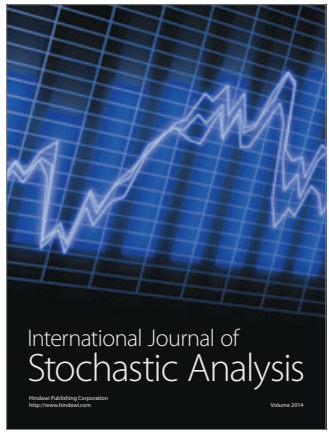

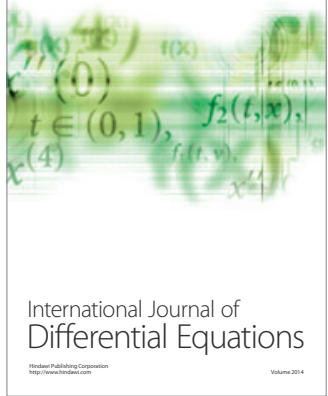
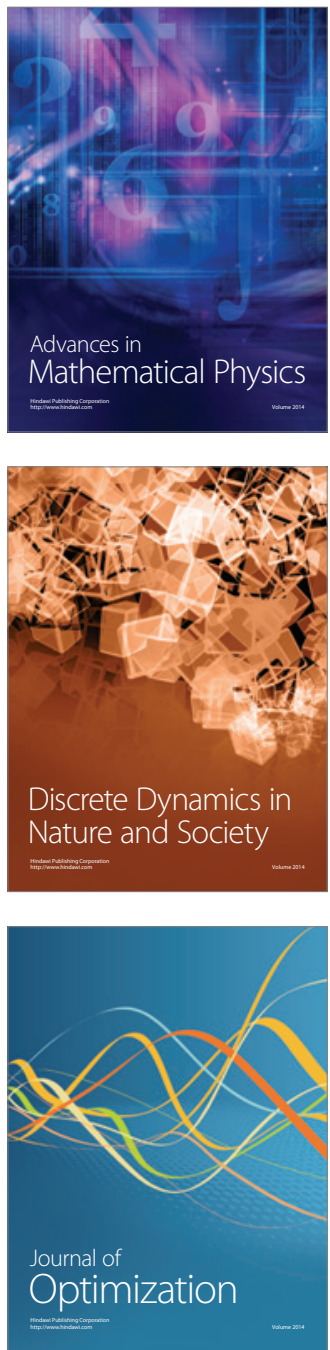